\title{
RTP801/REDD1 contributes to neuroinflammation severity and memory impairments in Alzheimer's disease
}

\author{
Leticia Pérez-Sisqués (iD ${ }^{1}$, Anna Sancho-Balsells ${ }^{1,2,3}$, Júlia Solana-Balaguer ${ }^{1}$, Genís Campoy-Campos ${ }^{1}$, Marcel Vives-Isern ${ }^{1}$, \\ Ferran Soler-Palazón ${ }^{1}$, Marta Anglada-Huguet ${ }^{4,5}$, Miguel-Ángel López-Toledano ${ }^{6}$, Eva-Maria Mandelkow ${ }^{4,5}$, Jordi Alberch (iD ${ }^{1,2,3,7}$, \\ Albert Giralt $\mathbb{D}^{1,2,3,7,8^{\bowtie}}$ and Cristina Malagelada $\mathbb{D i D}^{1,3,8} \mathrm{~B}^{凶}$
}

(c) The Author(s) 2021

RTP801/REDD1 is a stress-regulated protein whose upregulation is necessary and sufficient to trigger neuronal death. Its downregulation in Parkinson's and Huntington's disease models ameliorates the pathological phenotypes. In the context of Alzheimer's disease (AD), the coding gene for RTP801, DDIT4, is responsive to $A \beta$ and modulates its cytotoxicity in vitro. Also, RTP801 mRNA levels are increased in AD patients' lymphocytes. However, the involvement of RTP801 in the pathophysiology of AD has not been yet tested. Here, we demonstrate that RTP801 levels are increased in postmortem hippocampal samples from AD patients. Interestingly, RTP801 protein levels correlated with both Braak and Thal stages of the disease and with GFAP expression. RTP801 levels are also upregulated in hippocampal synaptosomal fractions obtained from murine 5xFAD and rTg4510 mice models of the disease. A local RTP801 knockdown in the 5xFAD hippocampal neurons with shRNA-containing AAV particles ameliorates cognitive deficits in 7-month-old animals. Upon RTP801 silencing in the 5xFAD mice, no major changes were detected in hippocampal synaptic markers or spine density. Importantly, we found an unanticipated recovery of several gliosis hallmarks and inflammasome key proteins upon neuronal RTP801 downregulation in the 5xFAD mice. Altogether our results suggest that RTP801 could be a potential future target for theranostic studies since it could be a biomarker of neuroinflammation and neurotoxicity severity of the disease and, at the same time, a promising therapeutic target in the treatment of AD.

Cell Death and Disease (2021)12:616; https://doi.org/10.1038/s41419-021-03899-y

\section{INTRODUCTION}

Alzheimer's disease (AD), the most common type of dementia affecting millions of people worldwide, is characterized by progressive cognitive impairment, typically beginning with memory deterioration and followed by executive dysfunction and language, visual and practical problems along with emotional and psychiatric symptoms [1, 2]. AD pathology starts in structures such as the hippocampus and the entorhinal cortex $[2,3]$, being the extracellular amyloid- $\beta(A \beta)$ plaques and intracellular tangles of abnormally hyperphosphorylated Tau the most representative $A D$ hallmarks [3]. $A \beta$ and phospho-Tau accumulation over the course of the disease impair synaptic plasticity, activate an inflammatory response, and compromise neuronal survival $[4,5]$.

Neuroinflammation has an active role in AD pathogenesis [6-9]. It is characterized by the activation of innate immune response due to misfolded or aggregated proteins, such as $A \beta$, that trigger microglial and astroglial activation and the consequent release of pro-inflammatory cytokines and chemokines. Inflammasomes, intracellular sensors of danger-associated molecular patterns (DAMPs), play an important role in triggering some of these inflammatory cascades. This response is intended to be beneficial at early stages, promoting $A \beta$ and neuron debris clearance, but if inflammation becomes chronic, it exacerbates neurodegeneration (reviewed in [10]).

The coding product of the DDIT4 gene is a stress-induced protein called RTP801/REDD1 [11-13]. RTP801 is a negative regulator of mTOR and Akt that is necessary and sufficient for neuron death in Parkinson's disease (PD) $[14,15]$. RTP801 is also elevated in PD [14, 16] and Huntington's disease (HD) human brains [17], suggesting an important role for this protein in both human diseases. In this line, RTP801 downregulation in the Substantia Nigra pars compacta restored motor-learning skills in a PD mouse model subjected to chronic stress [18]. Moreover, in the R6/1 mice, an HD mouse model, RTP801 silencing prevented motor impairment correcting, in turn, the alterations in synaptic plasticity [19]. Interestingly, RTP801 modulates synaptic plasticity in models of chronic unpredictable stress leading to depression [20], a co-morbid pathology associated with AD [21].

In the context of $A D, D D / T 4$ is an $A \beta$-responsive gene that modulates $A \beta$ cytotoxicity in vitro $[22,23]$. Moreover, both RTP801 mRNA and protein levels are increased in lymphocytes derived from $\mathrm{AD}$ patients [24]. DDIT4 also appears as one of the differentially

\footnotetext{
${ }^{1}$ Departament de Biomedicina, Facultat de Medicina i Ciències de la Salut, Institut de Neurociències, Universitat de Barcelona, Barcelona, Catalonia, Spain. ${ }^{2}$ Institut d'Investigacions Biomèdiques August Pi i Sunyer (IDIBAPS), Barcelona, Catalonia, Spain. ${ }^{3}$ Centro de Investigación Biomédica en Red sobre Enfermedades Neurodegenerativas (CIBERNED), Madrid, Spain. ${ }^{4}$ German Center for Neurodegenerative Diseases (DZNE), Bonn, Germany. ${ }^{5}$ CAESAR Research Center, Bonn, Germany. ${ }^{6}$ Neurelis, Inc., San Diego, CA, USA. ${ }^{7}$ Production and Validation Center of Advanced Therapies (Creatio), Faculty of Medicine and Health Science, University of Barcelona, Barcelona, Catalonia, Spain. ${ }^{8}$ These senior authors contributed equally: Albert Giralt, Cristina Malagelada. Edited by A. Verkhratsky. ${ }^{凶}$ email: albertgiralt@ub.edu; cristina.malagelada@ub.edu
}

Received: 19 November 2020 Revised: 25 May 2021 Accepted: 1 June 2021

Published online: 15 June 2021 
expressed genes in samples from the prefrontal cortex (PFC) from AD patients [25] and is one of the few genes of the mTOR pathway that might be affected by the ApoE genotype [26].

Here, we investigated whether RTP801 is involved in AD pathogenesis using human postmortem AD samples and transgenic animal models of the disease. We found that RTP801 is elevated in the hippocampus of $A D$ patients and its levels correlated with both Braak and Thal stages of the disease. Also, RTP801 was upregulated in hippocampal synaptosomal fractions from 5xFAD mice and in samples of the entorhinal cortex from rTg4510 mice (a mouse model of tauopathy). Hippocampal neuronal silencing of RTP801 expression in the 5xFAD mouse model prevented memory impairment and abrogated astro- and microgliosis. Altogether, our results suggest a putative role of RTP801 in the inflammatory response associated with $A D$ and frame RTP801 as a novel target in AD.

\section{RESULTS \\ Hippocampal RTP801 levels are increased in Alzheimer's disease patients and rodent models and correlate with neuropathological severity}

We first examined RTP801 protein levels in postmortem hippocampal human tissue (see Supplementary Table 1). In this region, total RTP801 levels were significantly increased in AD patients compared to controls (Fig. 1A, B). While RTP801 levels did not differ between $C T$ and $A D$ samples in synaptosomes, we detected reduced levels of the presynaptic protein SV2a in the synaptic compartment from $A D$ patients (Supplementary Fig. 1), as previously described [27]. We observed that RTP801 protein levels were also elevated in the crude synaptosomal compartment after correcting the synaptic loss by expressing protein levels relative to the expression of the presynaptic protein SV2a (Fig. 1C, D). We next performed correlation studies in $A D$ samples comparing RTP801 levels in the whole lysate with the Braak (Fig. 1E) and Thal (Fig. 1F) stages, respectively, for each case and we observed a significant positive correlation in both, suggesting that the neuropathological severity correlates with RTP801 levels. In addition, GFAP expression (a marker of astrogliosis [28]) was increased in AD patients (Fig. 1G) and positively correlated with RTP801 levels (Fig. 1H), reinforcing the idea of RTP801 levels as a marker of neuropathological severity.

We next analyzed the hippocampus of 5-month-old 5xFAD mice, an age at which they have an altered phenotype [29-32] comparable to the human samples. Total RTP801 levels were similar in wild-type (WT) and 5xFAD mice (Fig. 1l, J), whereas RTP801 levels in the synaptosomal fraction were higher in 5xFAD than in WT mice (Fig. $1 /-K)$. This result indicated a functional alteration of this protein depending on its subcellular localization, specifically in the synapse in an amyloid mouse model. Next, since RTP801 levels positively correlated with Braak stages in AD human samples (Fig. 1E) we sought potential similar changes in tauopathy rodent models. We took advantage of the rTg4510 model at 6 months of age, when the phenotype of this mouse model is evident [33]. Interestingly, total RTP801 levels were significantly increased in rTg4510 mice compared with WT mice (Fig. 1L, M), and similarly, RTP801 levels in the synaptosomal fraction were also higher in rTg4510 than in WT mice (Fig. 1L-N). Altogether, our results show that RTP801 levels were increased in AD patients, 5xFAD mice, and Tg4510 mice and that such increased levels positively correlated with the severity of neurofilament tangles distribution (Braak stages), progressive deposition of amyloid- $\beta$ (Thal stages), and astrogliosis.

Genetic normalization of hippocampal RTP801 levels in 5xFAD mice induces cognitive improvements related to associative and declarative memories

We observed that RTP801 levels were widely upregulated in human hippocampal postmortem samples from $A D$ patients, in a mouse model of tauopathy ( $\mathrm{Tg} 4150$ mice), and the synaptosomes of a mouse model of Amyloid- $\beta$ deposition (5xFAD mice). Thus, we next hypothesized that the normalization of RTP801 levels in the dorsal hippocampus of the 5xFAD mice could improve their memory deficits. To test this hypothesis, 6-month-old WT and 5XFAD mice received, in the dorsal hippocampus, a bilateral stereotactic injection of AAV expressing scramble shRNA (shCt) or shRNA against RTP801 (shRTP801) generating four groups of mice namely: WT shCt, WT shRTP801, 5xFAD shCt, and 5xFAD shRTP801.

Four weeks after injection, we performed a broad behavioral characterization as depicted in Fig. 2A. First, all mice were subjected to the plus-maze paradigm since this test is sensitive to several neurological disturbances in $5 \times$ FAD mice at this age $[34,35]$. In this test, the increased time spent in the open arms showed by $5 x F A D$ shCt mice compared with WT shCt mice was not corrected in 5xFAD shRTP801 mice (Fig. 2B). In the passive avoidance test, however, the 5xFAD shCt mice showed significantly lower latencies to step-through in the testing session compared with WT shCt mice (Fig. 2C). Interestingly, these differences were completely rescued in the 5xFAD shRTP801 mice (Fig. 2C). In the spontaneous alternation in a T-maze task (Fig. 2D, E), the arm preference and spontaneous alternation were evaluated $1 \mathrm{~h}$ after habituation. We found that the WT shCt, WT shRTP801, and 5xFAD shRTP801 mice explored significantly longer the novel arm than the familiar one whereas 5xFAD shCt mice showed no preference for any arm (Fig. 2D). Furthermore, WT shCt, WT shRTP801, and 5xFAD shRTP801 mice significantly alternated whereas $5 x F A D$ shCt mice did not (Fig. 2E). Thus, in the T-SAT 5xFAD shCt mice showed alterations in both variables, spontaneous alternation, and new context exploration, whereas 5xFAD shRTP801 mice showed significant restoration of these two parameters. Finally, we used the Morris water maze (MWM) to test possible alterations in associative and spatial learning. First, all groups showed normal muscular strength in the grid test (WT shCt 60.0 $\pm 0.0,5 x F A D$ shCt $53.36 \pm 3.636$, WT shRTP801 $60.0 \pm 0.0$ and 5xFAD shRTP801 $60.0 \pm$ 0.0 ; two-way ANOVA genotype effect: $F_{(1,41)}=1.047, P=0.3122$; two-way ANOVA shRNA effect: $\left.F_{(1,41)}=1.047, P=0.3122\right)$, suggesting the idea that potential changes in the MWM are not due to muscular strength deficits. Second, we found no differences in mice weight associated to RTP801 silencing although 5xFAD mice presented decreased body weight, as previously reported [36] (WT shCt $31.11 \pm 2.11,5 x F A D$ shCt $29.64 \pm 2.798$, WT shRTP801 31.09 \pm 3.093, and 5xFAD shRTP801 $29.10 \pm 2.935$; two-way ANOVA treatment effect: $F_{(1,62)}=0.1616, P=0.6891$; two-way ANOVA genotype effect: $\left.F_{(1,62)}=6.358, P=0.0143\right)$. Next, to exclude poor vision, altered motivation, and/or sensorimotor disabilities in 5xFAD groups of mice, all the animals were tested in the visible platform task (four trials per mouse). We found no differences between WT shCt, WT shRTP801, 5xFAD shCt, and 5xFAD shRTP801 mice in escape latencies during training with the visible platform (Fig. 2F). All the groups of mice improved rapidly across trials demonstrating that the task was acquired normally. When spatial learning was assessed using the hidden platform version of the MWM, we found that the performance improved significantly during training in WT shCt, WT shRTP801, and 5xFAD shRTP801 mice but not in 5xFAD shCt mice (Fig. 2G). Thus, WT shCt, WT shRTP801, and 5xFAD shRTP801 mice showed acquisition of spatial learning and memory, whereas 5xFAD shCt mice did not. Altogether, this set of results shows that 7.5-month-old 5xFAD mice suffer from deficits in associative and spatial learning and that the normalization of RTP801 levels in principal neurons of the CA1 and dentate gyrus (in the $5 \times$ FAD shRTP801 group) rescues these cognitive impairments.

Genetic normalization of hippocampal RTP801 levels in 5xFAD mice corrects core neuroinflammatory events

One week after behavioral testing, we sacrificed the animals to investigate the key events that could explain memory and 

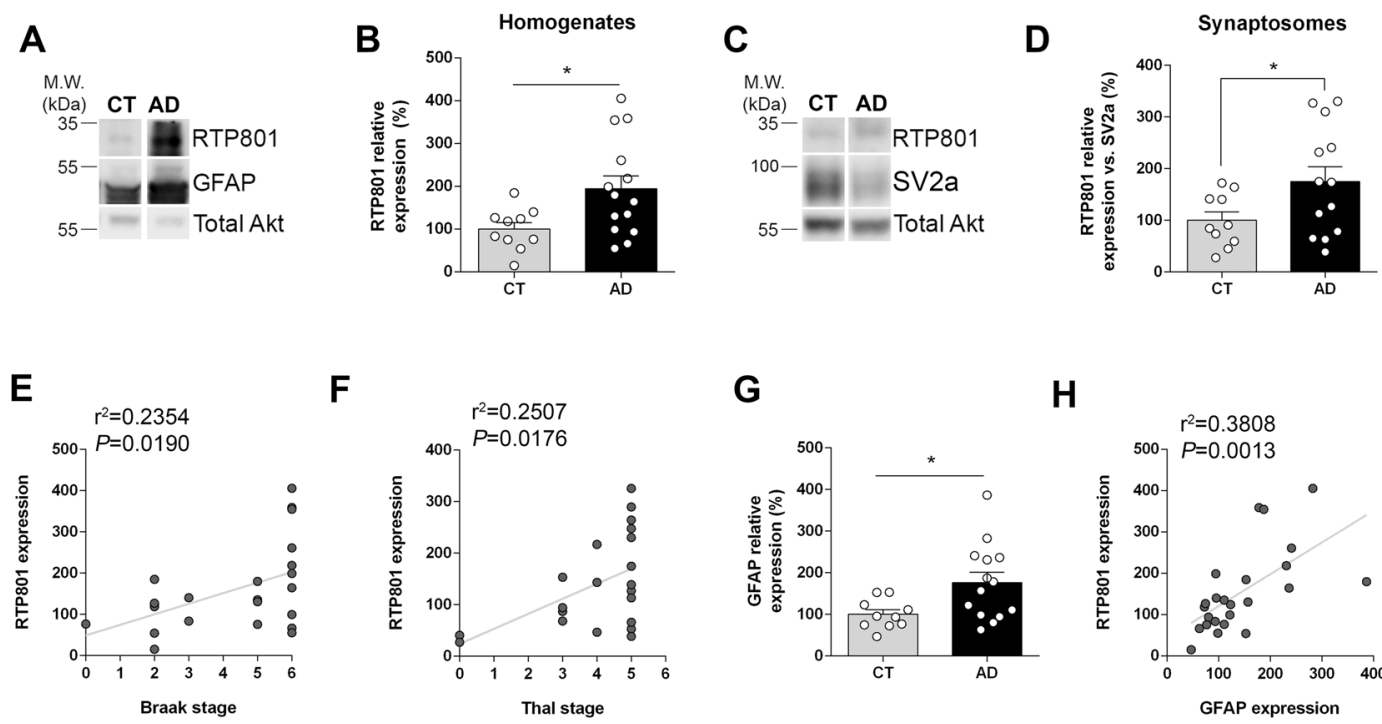

$\mathbf{F}$

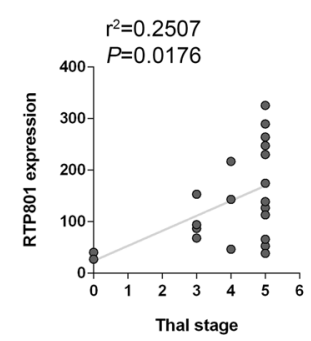

G

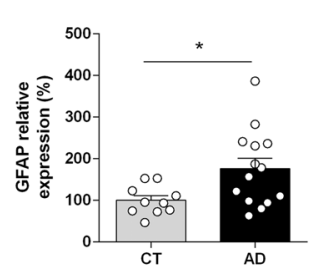

$\mathbf{H}$

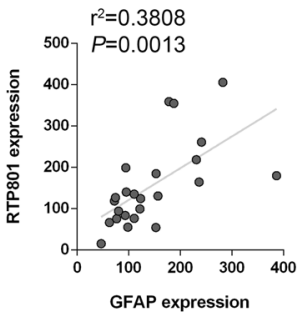

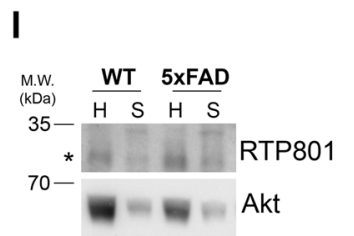
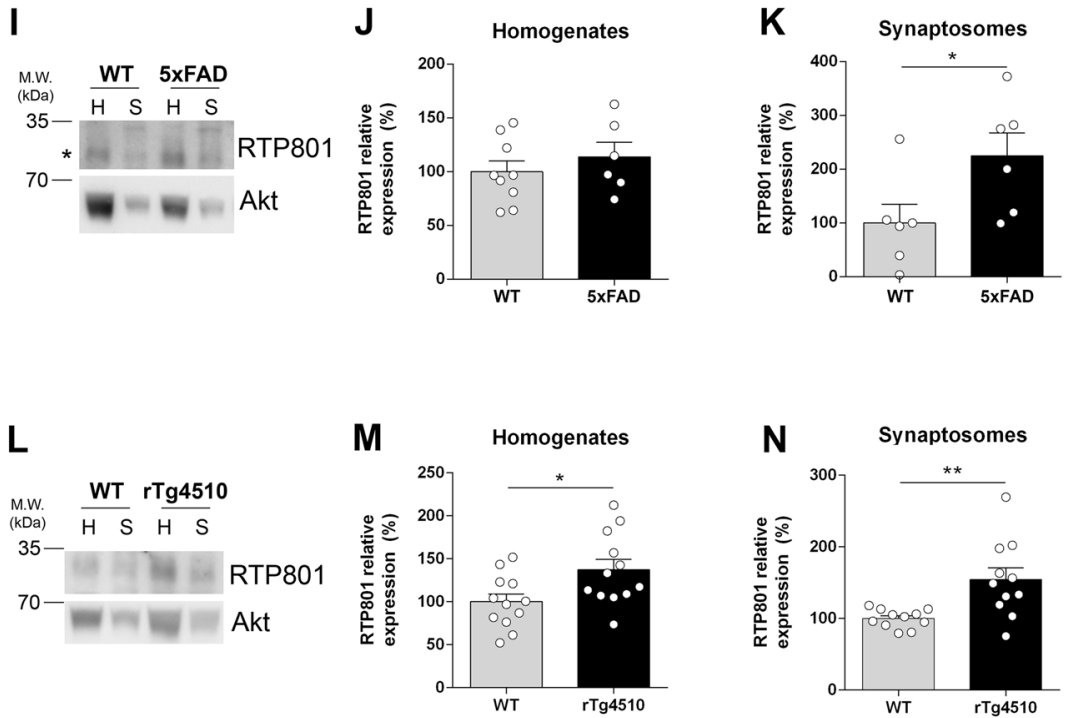

Fig. 1 RTP801 levels in human postmortem samples with Alzheimer's disease and 5xFAD and rTg4510 transgenic mice. A Immunoblotting for RTP801, GFAP, and Akt as a loading control in human postmortem hippocampal samples from controls (CT) and Alzheimer's disease (AD) patients. B Densitometric quantification of RTP801 results as in (A) for the hippocampus $\left(t_{18.75}=2.789, P=0.0118\right)$. C Immunoblotting for RTP801, SV2a, and Akt in the crude synaptosomal fractions from postmortem hippocampal samples from CT and AD patients. D Densitometric quantification of synaptic RTP801 levels normalized by SV2a levels $\left(t_{18.26}=2.254, P=0.0367\right)$. E Pearson's correlation analysis comparing RTP801 levels as in (B) with Braak stage per sample in AD patients $(P=0.019)$. $\mathbf{F}$ Pearson's correlation analysis comparing RTP801 levels as in (B) with Thal stage per sample in AD patients $(P=0.017)$. G Densitometric quantification of GFAP results as in (A) for the hippocampus $\left(t_{17.81}=2.843, P=0.0109\right)$. H Pearson's correlation analysis comparing GFAP levels as in (G) with RTP801 levels as in (B) per sample in AD patients $(P=0.0013)$. I Immunoblotting for RTP801 and Akt as a loading control in total $(\mathrm{H})$ and synaptosomal (S) hippocampal fractions from 5-month male and female WT and 5xFAD mice. Densitometric quantification in total (J) and synaptosomal (K) fractions of results as in (I) for RTP801 in 5-month-old mice (in J: $t_{13}=0.827, P=0.422$; in K: $t_{10}=2.254, P=0.047$ ). I Immunoblotting for RTP801 and Akt as a loading control in total $(\mathrm{H})$ and synaptosomal (S) hippocampal fractions from 6-month male and female WT and rTg4510 mice. Densitometric quantification in total $(\mathbf{M})$ and synaptosomal $(\mathbf{N})$ fractions of results as in (I) for RTP801 in 6-month-old mice (in $\mathbf{M}$ : $t_{22}=2.496, P$ $=0.0206$; in $\left.\mathbf{N}: t_{11.16}=3.284, P=0.0071\right)$. In $(\mathbf{A}, \mathbf{C}, \mathbf{I}$, and $\mathbf{L})$ molecular weight markers position is indicated in $\mathbf{k D a}$. In $\mathbf{B}, \mathbf{G}, \mathbf{J}, \mathbf{K}, \mathbf{M}$, and $\mathbf{N}$ data were normalized to Akt for each sample and expressed as a percentage of the mean of WT/controls, and means and SEM are indicated. Data are analyzed with the unpaired $t$ test in $\mathbf{B}, \mathbf{D}, \mathbf{G}, \mathbf{J}, \mathbf{K}, \mathbf{M}$, and $\mathbf{N}$ and with Pearson's correlation in $\mathbf{E}, \mathbf{F}$, and $\mathbf{H}$. In bands $\mathbf{B}, \mathbf{D}$, and $\mathbf{G},{ }^{*} P<0.05$ compared to CT. In $\mathbf{K}, \mathbf{M}$, and $\mathbf{N},{ }^{*} P<0.05$ and ${ }^{* *} P<0.01$ compared to WT. CT control, AD Alzheimer's disease, WT wild-type, $\mathrm{H}$ total homogenates, $\mathrm{S}$ synaptic homogenates.

learning improvement. We first confirmed widespread viral transduction in the dorsal hippocampus including the dentate gyrus (DG), the CA3, and the CA1 (Fig. 3A) and specific transduction of neuronal cells (Fig. 3B). We then confirmed by immunohistochemistry that the RTP801 levels were downregulated by the shRTP801 in the pyramidal neurons of the CA1 (Fig.
3C, D) and in the granular neurons of the DG (Fig. 3E, F), and found a $15 \%$ and a $40 \%$ decrease, respectively. These changes were further confirmed by western blot by evaluating RTP801 and GFP protein levels in the whole dorsal hippocampus (Fig. 3G, H), where we observed a $30-40 \%$ decrease in RTP801 levels in shRTP801injected mice. We next studied whether RTP801 silencing was 
A

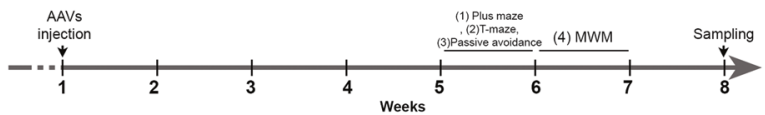

B

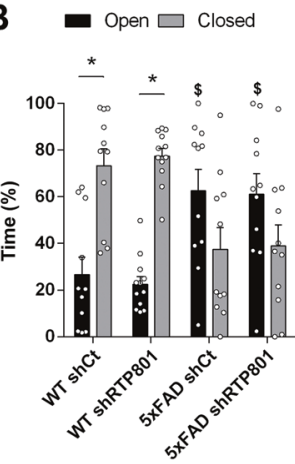

D
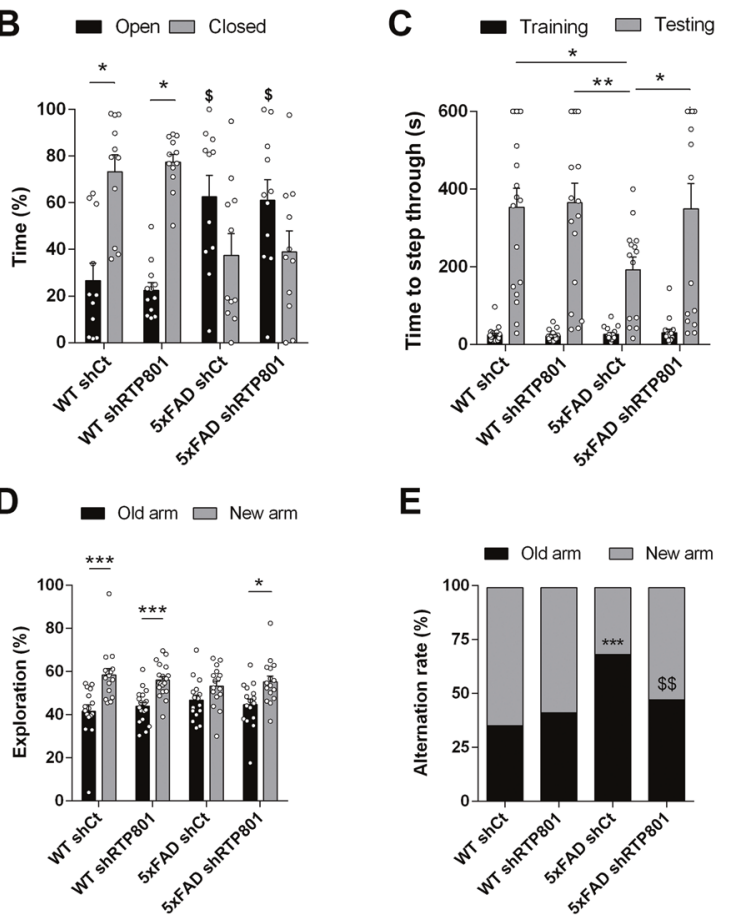

$E$

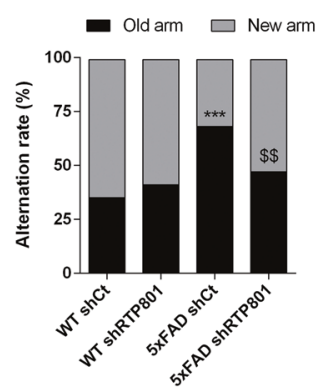

F $\rightarrow W T$ shCt $₫ 5 \mathrm{XFAD} \mathrm{shCt}$
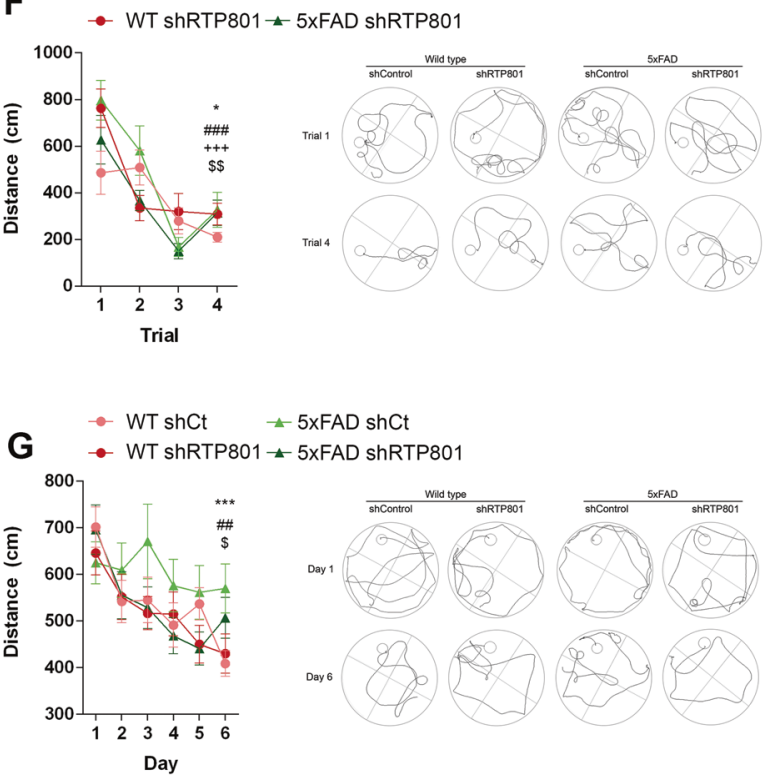

affecting plaque load in the dorsal hippocampus but no changes were found between 5xFAD shCt and 5xFAD shRTP801 animals (Supplementary Fig. 2). Next, we investigated whether RTP801 downregulation was affecting synaptic plasticity. Golgi-Cox staining showed no differences in spine density between the four groups (Fig. 4A, B). No significant differences were observed in the size of the head area of these spines either (Fig. 4C). However, silencing RTP801 in 5xFAD animals rescued the excessive neck length of their dendritic spines (Fig. 4D). Regarding the levels of synaptic proteins, we observed that RTP801
Fig. 2 Behavioral phenotype upon genetic inhibition of RTP801 levels in the dorsal hippocampus of 5xFAD male mice. As depicted in the scheme (A), AAVs expressing GFP-shCT (AAV-shCt) or GFPshRNA-RTP801 (AAV-shRTP801) were bilaterally injected in the dorsal hippocampus of 6-month-old WT males (WT shCt or WT shRTP801 groups) or 5xFAD males (5xFAD shCt or 5xFAD shRTP801 groups). Four weeks later, a battery of behavioral tests was performed. B Elevated plus maze. The time spent in the open arms was monitored for 5 min in 7-month-old WT shCt, WT shRTP801, 5xFAD shCt, and 5xFAD shRTP801 groups of mice. A genotype significant effect was detected (two-way ANOVA, $F_{(1,82)}=6.633, P=$ 0.0118 ). C Passive avoidance test. The latency (in seconds) to stepthrough was measured before (Training) and $24 \mathrm{~h}$ after (Testing) receiving an electric shock ( $2 \mathrm{~s} 1 \mathrm{~mA})$ in 7-month-old WT shCt, WT shRTP801, 5xFAD shCt, and 5xFAD shRTP801 groups of mice. A significant effect by groups was detected (two-way ANOVA, $\left.F_{(1,126)}=126.9, P=0.0001\right)$. D, E Spontaneous alternation learning in a T-maze. Spontaneous alternation learning was assessed by the T-SAT in 7-month-old WT shCt, WT shRTP801, 5xFAD shCt, and 5XFAD shRTP801 groups of mice for arms exploration (Arm preference effect: $F(1,126)=45.26, P<0.0001)$ and for spontaneous alternation rate (WT' shCt vs 5xFAD shCt: $\chi^{2}: 22.04, P<0.0001$ and 5xFAD shCt vs 5xFAD shRTP801: $\left.X^{2}: 9.148, P<0.01\right) 1 \mathrm{~h}$ after the training trial. $\mathbf{F}, \mathbf{G}$ Morris water maze. $\mathbf{F}$ The distance (in $\mathrm{cm}$ ) to reach the visible platform was monitored in a 4-trials session to evaluate potential visual or physical impairments in 7-month-old WT shCt, WT shRTP801, 5xFAD shCt, and 5xFAD shRTP801 groups of mice. Twoway ANOVA showed a significant general time effect in this procedural version of the MWM $\left(F_{(3,192)}=35.12, P<0.0001\right)$ and post hoc (Tukey's test) multiple comparisons indicated that all groups significantly improved their latencies in trial 4 compared to trial 1 (see graph). G The distance (in $\mathrm{cm}$ ) to reach the hidden platform was monitored in a daily 4-trials session performed for 6 days to evaluate spatial learning in 7-month-old WT shCt, WT shRTP801, 5xFAD shCt, and 5xFAD shRTP801 groups of mice. Twoway ANOVA showed a significant general time effect in this spatial version of the MWM $\left(F_{(5,320)}=9.413, P<0.0001\right)$ and post hoc (Tukey's test) multiple comparisons indicated that all groups significantly improved their latencies to reach the hidden platform on the day of training 6 compared to the day of training 1, except the 5xFAD shCt group who showed no significant differences comparing its latencies on day 6 with those on day 1 (see graph). The number of mice in F, G, WT shCt $(n=16)$, WT shRTP801 $(n=18)$, 5xFAD shCt $(n=16)$, and 5xFAD shRTP801 $(n=16)$. Data are means \pm SEM. In B, C, and D, a two-way ANOVA with Bonferroni's post hoc test was performed: ${ }^{*} P<0.05,{ }^{* *} P<0.01,{ }^{* * *} P<0.001$ when comparing open vs closed or training vs testing or old arm vs new arm on each genotype. In B, ${ }^{5} \mathrm{P}<0.05$ vs. WT shCt open arm. In E, Chi-square $\left(X^{2}\right)$ test was performed in pair comparisons: ${ }^{* * *} P<0.001$ compared with WT shCt and ${ }^{\$} P<0.01$ compared with $5 x$ FAD shCt. In $\mathbf{F}$, ${ }^{*} P<$ 0.05 comparing trials 1 vs 4 in WT shCt, \#\#\# $P<0.001$ comparing trials 1 vs 4 in WT shRTP801, ${ }^{+++} P<0.001$ comparing trials 1 vs 4 in 5 XFAD shCt and ${ }^{\$} P<0.01$ comparing trials 1 vs 4 in 5xFAD shRTP801. In G, ${ }_{* * *} P<0.001$ comparing days 1 vs 6 in WT shCt, ${ }^{\# \#} P<0.01$ comparing days 1 vs 6 in WT shRTP801 and ${ }^{\$} P<0.05$ comparing days 1 vs 6 in 5xFAD shRTP801.

expression normalization in the 5xFAD mice did not prevent the loss of synaptophysin, previously reported to be decreased in $5 \times F A D$ mice at this age $[29,35]$ (Fig. 4E, F). Levels of full-length TrkB receptor (TrkB.FL), another neuronal marker that participates in $A D$ pathology [37], were not affected either (Fig. 4G, H). However, the levels of the truncated TrkB (TrkB.T1), an isoform of the receptor with important regulatory effects in astrocytes and associated with toxicity and inflammation [38-40], were corrected after knocking down RTP801 in the 5xFAD hippocampus (Fig. 4l, J).

In line with that, we observed that RTP801 levels were also heavily upregulated in the astrocytes of the CA1 in 5xFAD mice compared to WT mice. Although astrocytes were not transduced with our neuron-specific AAVs serotype (Fig. 3B), knocking down RTP801 in 5xFAD mice reduced astroglial RTP801 levels similar to 
A
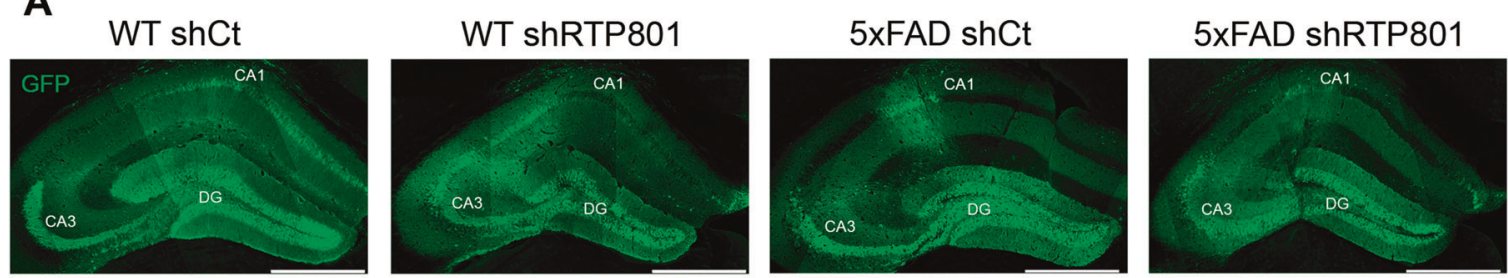

B
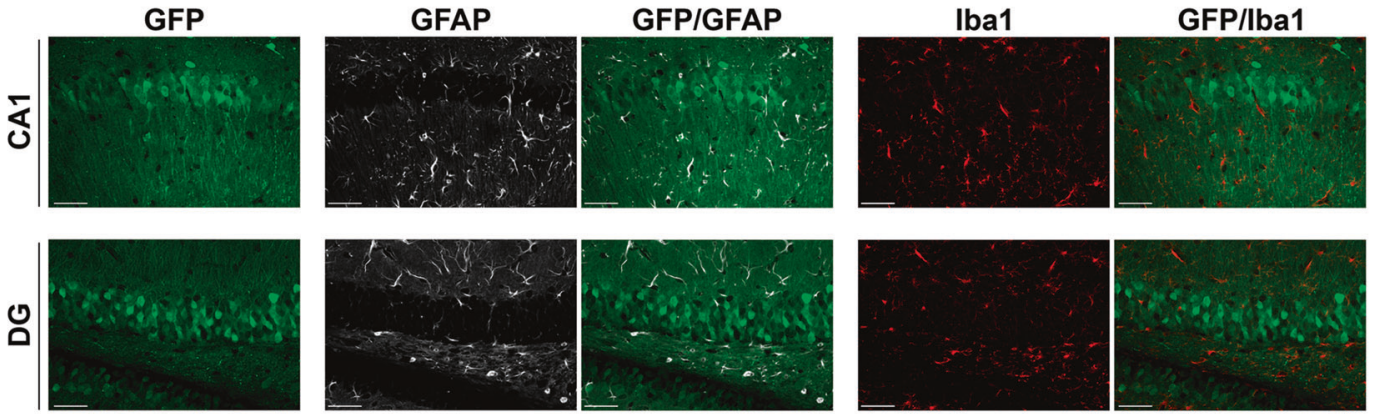

C
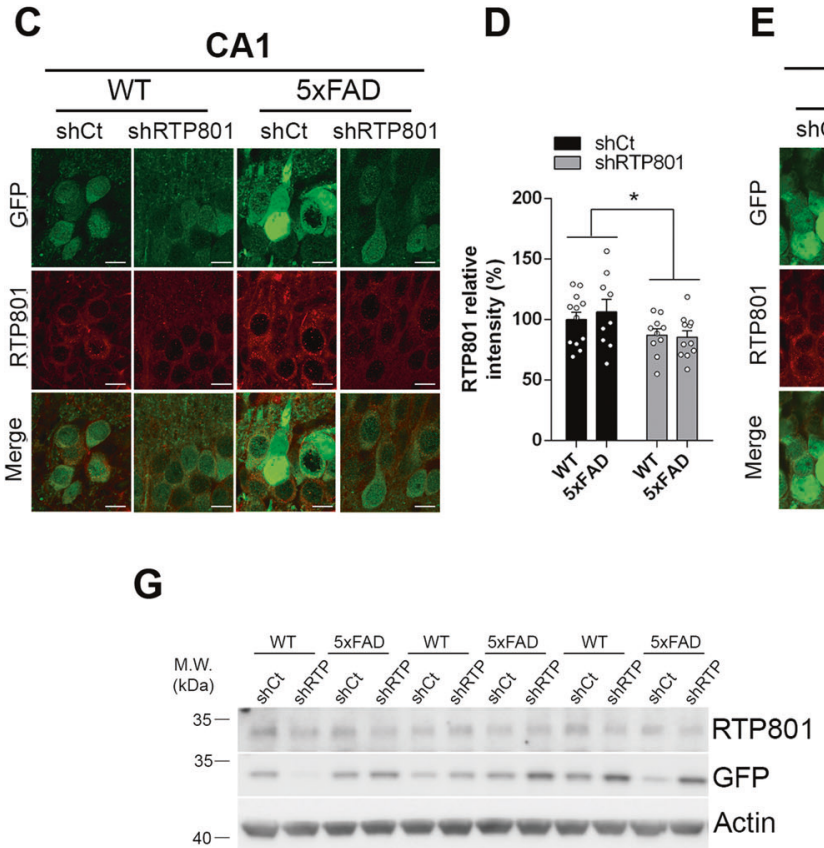

G

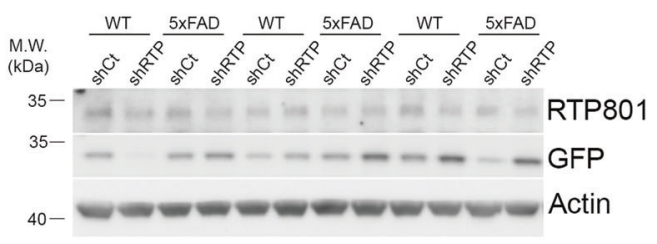

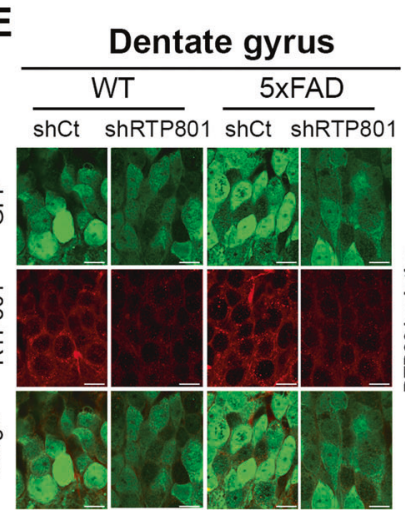

F

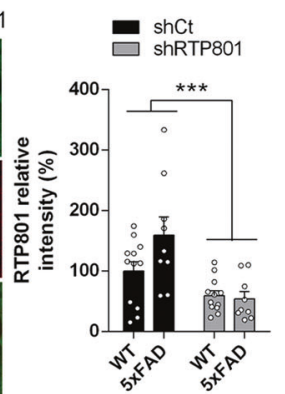

H

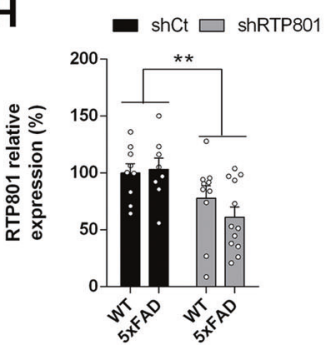

Fig. 3 Genetic inhibition of RTP801 levels in the dorsal hippocampus of 5xFAD mice. A Representative dorsal hippocampi from WT shCt, WT shRTP801, 5xFAD shCt, and 5xFAD shRTP801 mice 8 weeks after the injection, GFP fluorescence (green) was detected in the entire dorsal hippocampus. B Neuronal transduction specificity with AAV 2/8. Representative CA1 and DG images from dorsal hippocampus where transduced cells (GFP +, green), astrocytes (GFAP +, white), and microglial cells (Iba1 +, red) are depicted. Transduced cells were found in the CA1 pyramidal layer and DG granular layer. No glial cells were transduced. C Representative immunostained CA1 pyramidal cells stained for GFP (green) and RTP801 (red). D Immunoreactivity quantification of the RTP801 levels in pyramidal neurons of the CA1 in the four groups is shown (treatment effect: $F_{(1,38)}=6.197, P=0.0173$ ). E Representative immunostained granular cells of the DG stained for GFP (green) and RTP801 (red). F Immunoreactivity quantification of the RTP801 levels in granular neurons in the four groups is shown (treatment effect: $\left.F_{(1,38)}=17.73, P=0.0002\right)$. G Representative immunoblots showing the levels of RTP801 relativized with respect to GFP/actin ratio levels as the corresponding loading controls in dorsal hippocampus extracts from WT shCt, WT shRTP801, 5xFAD shCt, and 5xFAD shRTP801 groups of mice. $\mathbf{H}$ The histogram represents the protein expression expressed as a percentage of WT shCt (treatment effect: $\left.F_{(1,35)}=11.00, P=0.0021\right)$. In (G), molecular weight markers position is indicated in $\mathrm{kDa}$. All data are shown as the mean $\pm \mathrm{SEM}$. All data were analyzed by two-way ANOVA followed by Bonferroni's post hoc test: ${ }^{*} P<0.05$, ${ }^{* *} P<0.01$, ${ }^{* *} P<0.001$. DG dentate gyrus. Scale bars: A 500 microns; B 50 microns; D 10 microns.

WT mice injected with shCt AAVs (Fig. 5A, B), suggesting an RTP801-mediated effect from neurons to glial cells in the 5xFAD mice. Reinforcing this idea, 5xFAD mice transduced with shCt displayed an increased number of astrocytes per field (Fig. 5A-C) and increased GFAP-immunoreactivity intensity (Fig. 5A-D) compared to WT shCt whereas 5xFAD mice transduced with shRTP801 AAVs showed a complete rescue of these two parameters (Fig. 5C, D). Finally, a similar rescue effect was 
A

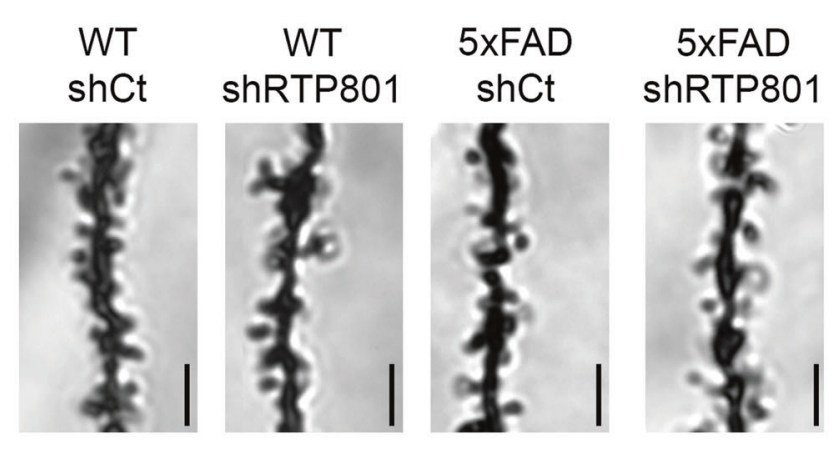

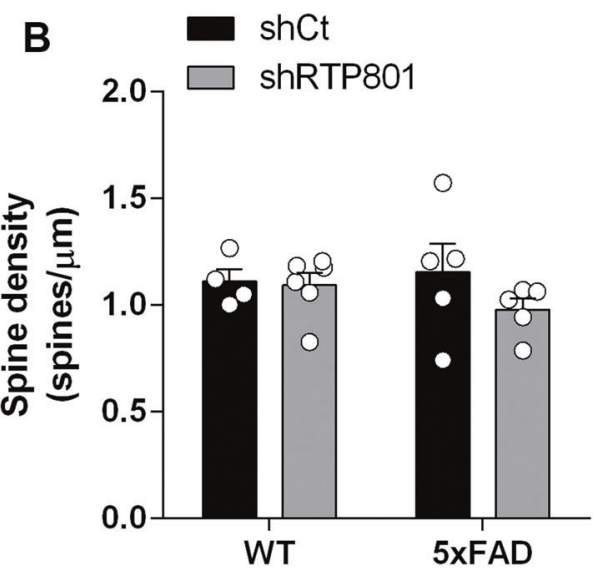
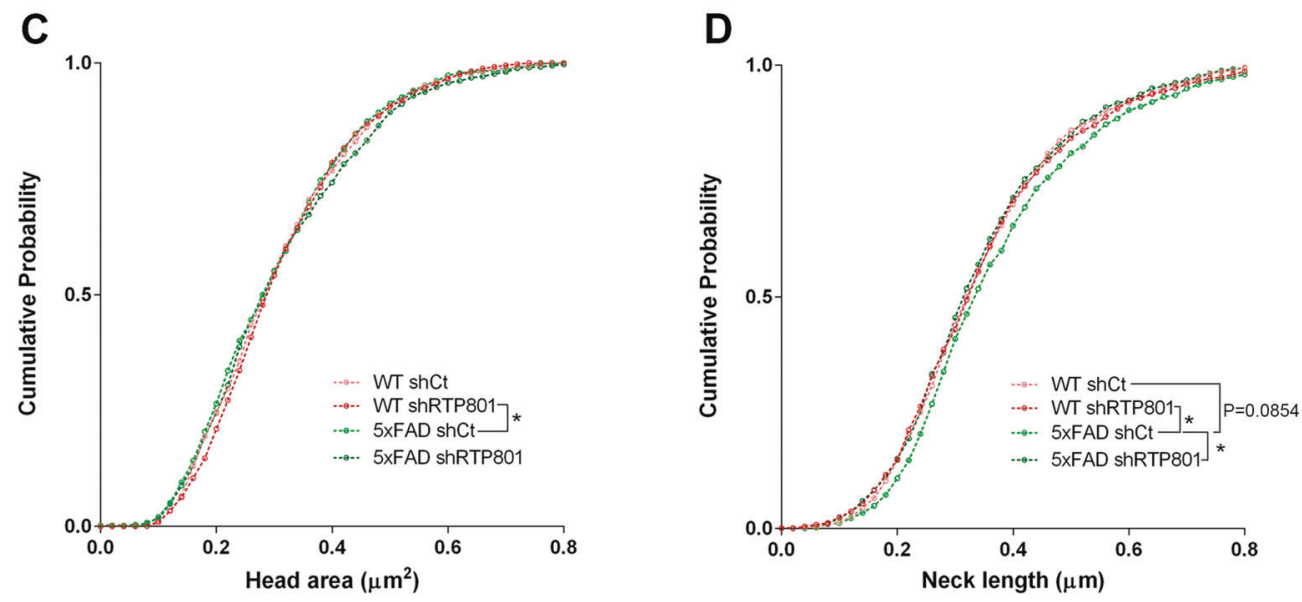

E

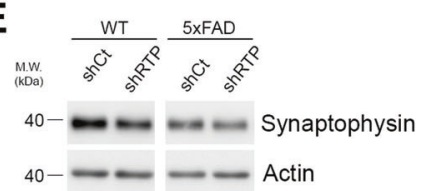

$\mathbf{F}$

$\square$ shCt
$\square$ shRTP801

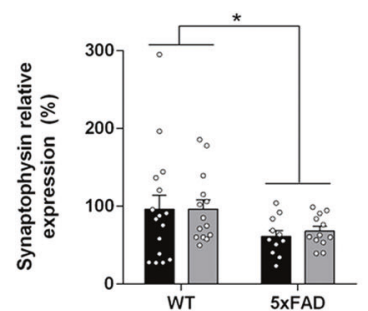

G

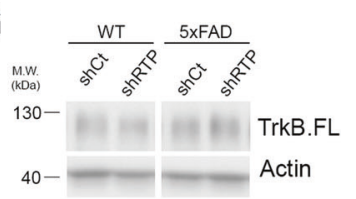

$\mathbf{H} \boldsymbol{m}$ shct

$\square$ shRTP801

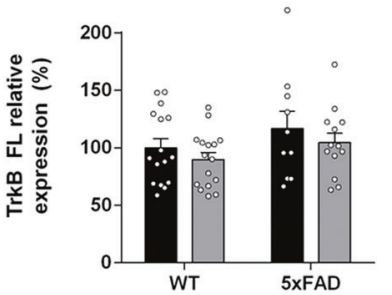

I

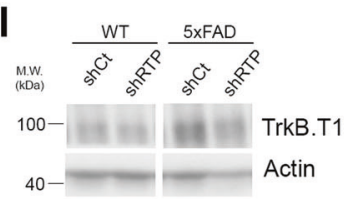

$\mathbf{J}$
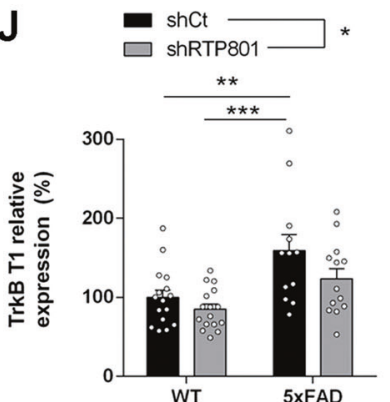

Fig. 4 Spine density and morphology and synaptic markers in the dorsal hippocampus in shRTP801-injected mice. A Golgi-Cox-stained proximal apical dendrites of CA1 stratum radiatum pyramidal neurons. Scale bar, $2 \mu \mathrm{m}$. B Quantification of spine density in dendrites as in (A). Data show mean spine density from 20 dendrites per animal (two-way ANOVA treatment effect: $F_{(1,16)}=1.297, P=0.2716$ ). C, D Cumulative probability of spine head area (C) and spine neck length (D) in 10-15 dendrites per animal from four to six animals per group. Distributions were compared with the Kolmogorov-Smirnov test. ${ }^{*} P<0.05$. No differences were obtained in spine head diameter. Neck length, WT shCt vs. $5 x F A D$ shCt, $\mathrm{D}=0.06287 P=0.0854$; WT shRTP801 vs. 5xFAD shCt, $\mathrm{D}=0.07492, P=0.0224 ; 5 x \mathrm{FAD}$ shCt vs. $5 x \mathrm{FAD}$ shRTP801,D $=0.07158, P=$ 0.0467. E-J Immunoblotting for the synaptic markers synaptophysin (E), TrkB.FL (G) and TrkB.T1 (I) and actin as the loading control. Densitometric quantification of synaptophysin (F) (genotype effect: $\left.F_{(1,49)}=5.538, P=0.0227\right)$, TrkB.FL $(\mathbf{H})\left(\right.$ treatment effect: $F_{(1,51)}=1.558, P=$ $0.2176)$ and TrkB.T1 (J) (treatment effect: $\left.F_{(1,53)}=4.281, P=0.0434\right)$ as in $(\mathbf{E}, \mathbf{G}$, and $\mathbf{I})$. In $(\mathbf{E}$, $\mathbf{G}$, and $\mathbf{I})$ molecular weight markers position is indicated in $\mathrm{kDa}$. All data are shown as the mean $\pm \mathrm{SEM}$. All data were analyzed by two-way ANOVA followed by Bonferroni's post hoc test: ${ }^{*} P<0.05,{ }^{* *} P<0.01,{ }^{* * *} P<0.001$. 

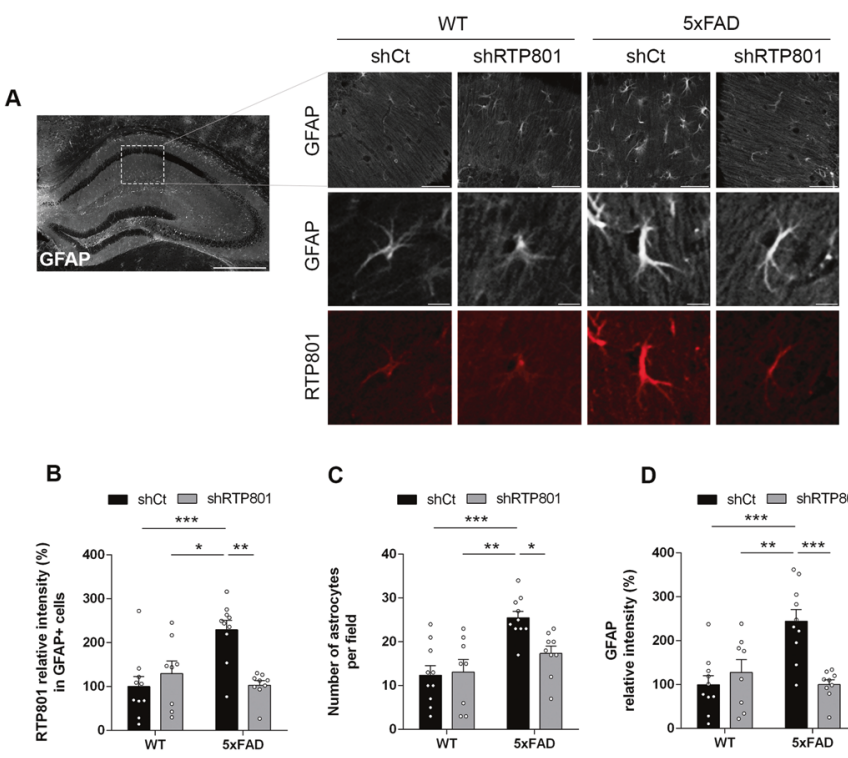

C
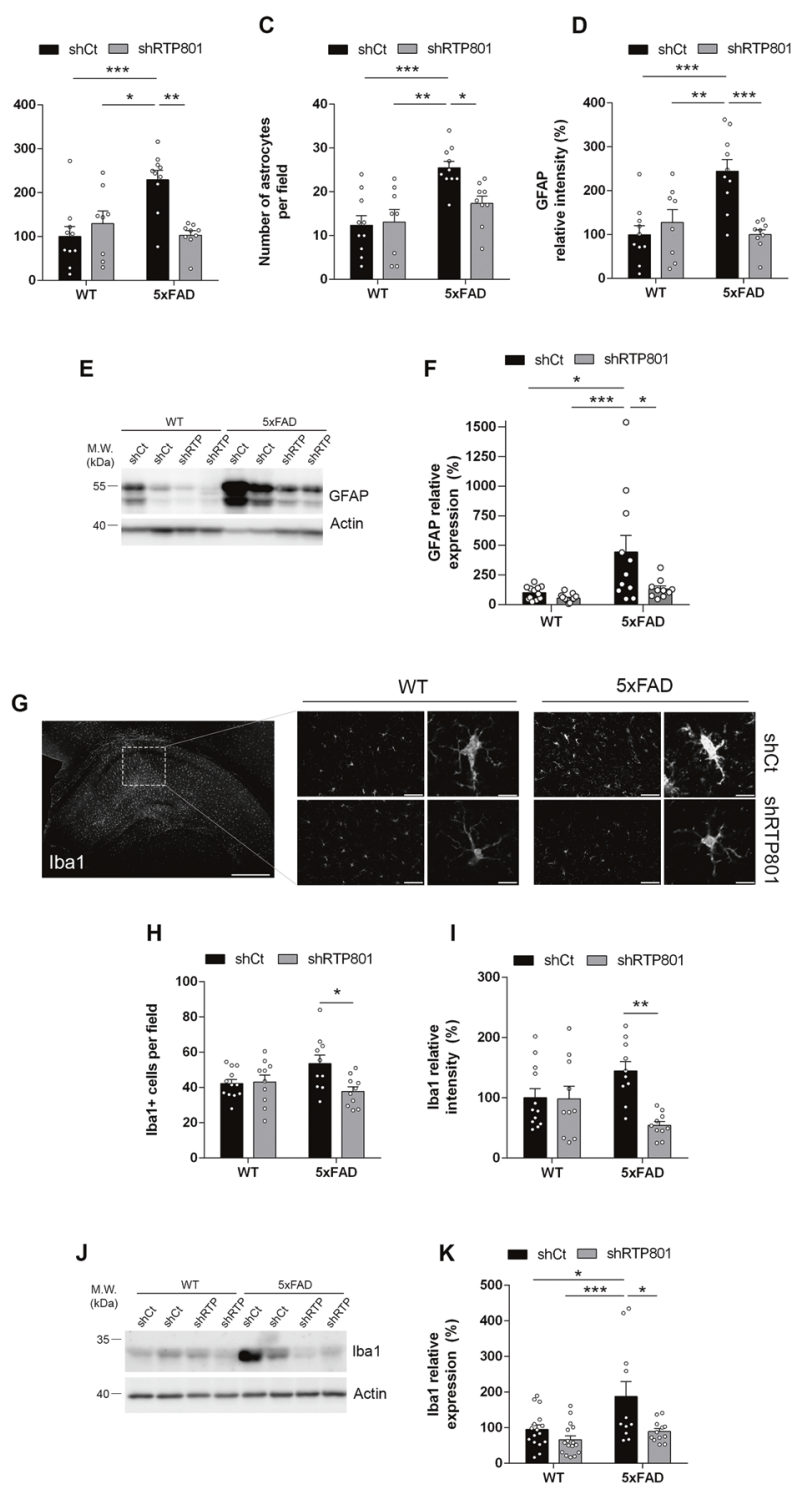

observed by western blot evaluating total GFAP levels in the entire dorsal hippocampus (Fig. 5E, F) strengthening the idea that normalization of RTP801 levels in hippocampal principal neurons restores and ameliorates the astrogliosis processes associated with the 5xFAD mutations.

To further gain insight into the neuroinflammatory processes mediated by RTP801 in the 5xFAD mice, we then evaluated microglial processes in the same animals. Microgliosis or changes in microglial states have been described in neurodegenerative disorders such as AD [41]. To do so, we first assessed the number
Fig. 5 Genetic inhibition of hippocampal RTP801 levels and neuroinflammatory markers. A Representative GFAP immunofluorescence microscopy imaging in the dorsal hippocampus (left panel, scale bar, 500 microns) and representative GFAP and RTP801 labeling in inset images from the CA1 in 7.5-month-old WT shCt, WT shRTP801, 5xFAD shCt, and 5xFAD shRTP801 groups of mice (right panels, scale bars 100 microns and 10 microns, respectively). B Quantification of RTP801 levels (IOD intensity) in CA1 GFAPpositive cells from a in the four groups (treatment effect: $F_{(1,33)}=$ 4.995, $P=0.0323$; interaction effect: $\left.F_{(1,33)}=13.11, P=0.001\right)$. C Quantification of GFAP-positive cell density in the CA1 from $\mathbf{A}$ in the four groups (treatment effect: $F_{(1,33)}=3.259, P=0.0802$; Interaction effect: $\left.F_{(1,33)}=4.653, P=0.0384\right)$. D Quantification of GFAP relative intensity (\% respect to WT shCt) in the CA1 from $\mathbf{A}$ in the four groups (treatment effect: $F_{(1,33)}=6.445, P=0.0160$; interaction effect: $\left.F_{(1,33)}=14.11, P=0.0007\right)$. E Immunoblotting for GFAP and actin as a loading control in the hippocampus of 7.5month-old WT shCt, WT shRTP801, 5xFAD shCt, and 5xFAD shRTP801 groups of mice. $\mathbf{F}$ Densitometric quantification of GFAP results as in (e) for the hippocampus (treatment effect: $F_{(1,44)}=9.941, P=0.0029$; genotype effect: $\left.F_{(1,44)}=7.085, P=0.0108\right)$. G Representative Iba1 immunofluorescence microscopy imaging in the dorsal hippocampus (left panel, scale bar, 500 microns) and representative lba1 labeling in inset images from the CA1 in 7.5-month-old WT shCt, WT shRTP801, 5xFAD shCt, and 5xFAD shRTP801 groups of mice (right panels, scale bars; 100 and 10 microns respectively). $\mathbf{H}$ Quantification of Iba1-positive cell density in the CA1 from $\mathbf{G}$ in the four groups (treatment effect: $F_{(1,38)}=4.952, P=0.0321$; interaction effect: $\left.F_{(1,38)}=6.210, P=0.0172\right)$. I Quantification of Iba1 relative intensity (\% respect to WT shCt) in the CA1 from $\mathbf{G}$ in the four groups (treatment effect: $F_{(1,38)}=8.821, P=0.0051$; interaction effect: $\left.F_{(1,38)}=8.211, P=0.0067\right)$. J Immunoblotting for Iba1 and actin as a loading control in the hippocampus of 7.5-month-old WT shCt, WT shRTP801, 5xFAD shCt, and 5xFAD shRTP801 groups of mice. $\mathbf{K}$ Densitometric quantification of $\mid \mathrm{ba} 1$ results as in (J) for the hippocampus (treatment effect: $F_{(1,51)}=10.16, P=0.0024$; genotype effect: $\left.F_{(1,51)}=8.448, P=0.0054\right)$. Data are means \pm SEM. In all panels, two-way ANOVA with Bonferroni's post hoc test was performed: ${ }^{*} P<0.05,{ }^{* *} P<0.01$, ${ }^{* *} P<0.001$.

of Iba1-positive microglial cells in the CA1, which trended to increase in 5xFAD shCt mice, whereas in 5xFAD mice transduced with shRTP801 this number was significantly reduced (Fig. 5G, H). Similar results were observed when the intensity of Iba1immunoreactivity was evaluated, showing a trend to increase in 5XFAD shCt and the same parameter was significantly reduced in 5xFAD mice transduced with shRTP801 (Fig. 5G-I). Finally, global changes on lba1 levels in the entire dorsal hippocampus were also evaluated by western blot and we observed a significant increase of lba1 protein levels in the $5 x F A D$ shCt mice compared with WT shCt mice whereas this increase was completely rescued in $5 x F A D$ shRTP801 mice (Fig. 5J, K).

We observed that the effect of silencing RTP801 in the 5xFAD mice was mTOR-independent (Supplementary Fig. 3) since we did not observe any changes in either the phosphorylation levels of mTOR or phospho-S6RP, as an mTORC1 kinase activity readout (Supplementary Fig. 3a-d). We only saw a significant increase of the levels of phospho-Ser473 Akt, as an mTORC2 substrate, in the 5xFAD animals that were not significantly ameliorated upon RTP801 silencing (Supplementary Fig. 3e, f).

Hence, to deepen the investigation of the mechanism by which silencing RTP801 in neurons diminished the inflammatory response in the 5xFAD model, we assessed the levels of inflammasome receptors and their components. We detected that elevated levels of NLRP1, which is mainly expressed in neurons [42], as pro-form (Fig. 6A, B) and auto-proteolytic fragment (cleaved) (Fig. 6C, D), are normalized by RTP801 silencing in neurons. The same results were seen for NLRP3, a receptor generally expressed in astrocytes and microglia 

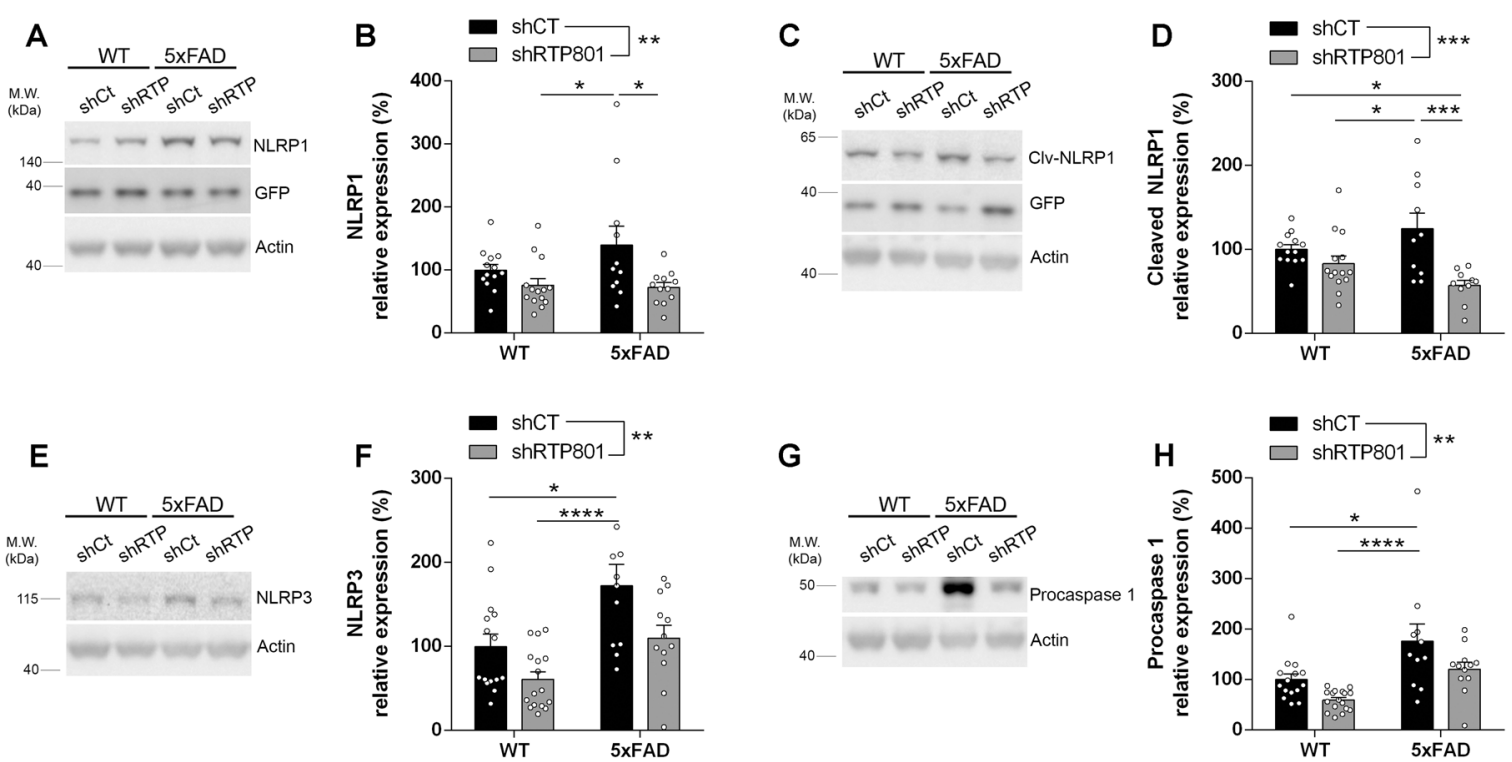

Fig. 6 Genetic inhibition of RTP801 levels in the dorsal hippocampus reduces the levels of inflammasome components NLRP1 and NLRP3 and procaspase 1. A, C Immunoblottings for NLRP1, cleaved NLRP1, and GFP as loading control for transduced neurons in the dorsal hippocampus of 7.5-month-old WT shCt, WT shRTP801, 5xFAD shCt, and 5xFAD shRTP801 groups of mice. B, D Densitometric quantification of NRLP1 and cleaved NLRP1 results as in $(\mathbf{A}, \mathbf{C})$ for the hippocampus (NLRP1 treatment effect: $F_{(1,46)}=8.714, P=0.0050$, interaction: $F_{(1,46)}=$ 1.932, $P=0.1713$; cleaved NLRP1 treatment effect: $F_{(1,46)}=15.84, P=0.0003$, interaction: $\left.F_{(1,46)}=5.702, P=0.0214\right)$. E, G Immunoblottings for NLRP3 and procaspase 1 and actin as loading control in the dorsal hippocampus of 7.5-month-old WT shCt, WT shRTP801, 5xFAD shCt, and 5xFAD shRTP801 groups of mice. F, H Densitometric quantification of NRLP3 and procaspase 1 results as in (E, G) for the hippocampus (NLRP3 treatment effect: $F_{(1,50)}=10.28, P=0.0023$, genotype effect: $F_{(1,50)}=14.72, P=0.0004$; procaspase 1 treatment effect: $F_{(1,51)}=8.438, P=$ 0.0054 , genotype effect: $\left.F_{(1,51)}=17.29, P=0.0001\right)$. Data are means \pm SEM. In all panels two-way ANOVA with Bonferroni's post hoc test was performed: ${ }^{*} P<0.05,{ }^{*} P<0.01,{ }^{* * *} P<0.001$.

(Fig. 6E, F). Levels of procaspase 1, a common effector for NLRP1 and 3, were also normalized after silencing RTP801 in neurons (Fig. $6 \mathrm{G}, \mathrm{H})$. On the contrary, we did not observe significant changes in cleaved caspase 1 ( $\mathrm{p} 20$ ) levels or IL-1 $\beta$ production (Supplementary Fig. 4a-e). AIM2 levels, another inflammasome receptor activated by DNA damage, were sensitive to RTP801 silencing (Supplementary Fig. 4d, f).

Altogether, our results suggest that silencing RTP801 levels in hippocampal principal neurons induces, in turn, a correction of aberrantly increased levels of RTP801 in astrocytes and significant amelioration of general neuroinflammatory processes in 5xFAD mice by regulating the NLRP1 and NLRP3 inflammasomes.

\section{DISCUSSION}

Here, we found that the stress-induced protein RTP801 is upregulated in the hippocampus from both human AD patients and in the $5 \times \mathrm{XFD}$ and tauopathy murine models of AD. Moreover, RTP801 levels in human postmortem hippocampal samples correlated with Braak and Thal stages that classify disease progression and severity. Remarkably, in these samples, RTP801 levels also correlated with GFAP expression, as a marker of astrogliosis. Indeed, RTP801 expression abrogation in the 5xFAD hippocampus prevented the cognitive decline associated with $A \beta$ deposition. Interestingly, RTP801 downregulation was associated with an anti-inflammatory effect with a dramatic decrease of astrogliosis, microgliosis, and a reduction of AIM2, NLRP1, and NLRP3 inflammasome sensor proteins.

In human postmortem hippocampal samples, we observed that RTP801 levels were significantly elevated in whole-brain lysates and the synaptic compartment. This is in line with previous results in other neurodegenerative diseases such as PD [14, 16] and HD $[17,19]$, supporting the crucial role of RTP801 in neurodegeneration. Moreover, the correlation of RTP801 levels with the Braak and the Thal stages suggests that RTP801 could be considered as a biomarker in AD. Since Damjanac et al. also found RTP801 mRNA and protein levels elevated in lymphocytes derived from $A D$ patients [24], we speculate that RTP801 could be treated as a systemic responsive protein in AD pathology and/or progression.

We found that RTP801 was elevated in compromised structures of the 5xFAD model and the rTg4510 tauopathy mouse models, in the hippocampus and the entorhinal cortex, respectively. This result suggests that the upregulation of RTP801 is common in the $A \beta$ and the phospho-Tau toxic signaling cascades, in line with the positive correlation that we observed between RTP801 levels and Thal and Braak stages. Indeed, RTP801 was differentially elevated in the crude synaptosomal compartment from both transgenic models, suggesting a specific dysregulation of RTP801 at the synapses. RTP801 has been recently described to be significantly elevated in human HD and R6/1 mice striatal synaptic preparations [19]. These results suggest that altered synaptic RTP801 levels could be a common molecular mechanism in neurodegenerative diseases.

5xFAD mice display severe impairments in associative and spatial learning $[35,43]$ as occurs in human patients with AD [4447]. Behavioral testing confirmed a remarkable rescue of cognitive skills by specifically silencing RTP801 in principal neurons (pyramidal and granular neurons) of the 5xFAD hippocampus. Hence, RTP801 mediates the loss of associative and declarative memories. However, this rescue did not involve changes in plaque load and size, suggesting that RTP801 function in AD does not modulate $A \beta$ homeostasis.

The plastic role of RTP801 was first described by Ota and colleagues in a model of depression induced by chronic unpredicted stress, where RTP801 knockout mice were more resilient to stress and showed much less dendritic spine loss [20]. A similar role was corroborated in an A53T a-synuclein mouse model of PD under chronic restraint stress [18]. Recently, we found that RTP801-mediated motor-learning deficits in the R6/1 mouse model of HD [19]. 
Strikingly, RTP801 knockdown did not affect spine density but prevented the excessive spine neck length found in the 5xFAD. Longer spine necks have been associated with a decrease in the amplitude in EPSCs recordings, while shorter spine necks are associated with increased synaptic strength [48-50]. Hence, this morphological feature due to RTP801 silencing in the 5xFAD could be contributing to the prevention-cognitive deficits in this model.

The implication of RTP801 in the activation of inflammatory pathways has been addressed in several in vitro and in vivo studies [51-54]. However, the putative role of the protein in the inflammatory pathways in the nervous system had never been studied. Here, we found that RTP801 silencing reduced TrkBtruncated receptor isoform $\mathrm{t} 1$ (TrkB.T1). TrkB.T1 is present in senile plaques, elevated in $A D$ brain samples and its overexpression in a mouse model of $A D$ aggravates its memory impairment $[38,55,56]$. Interestingly, TrkB.T1 is the only TrkB isoform expressed by astrocytes [57] and regulates gliosis and the inflammatory response $[58,59]$. Hence, these results suggest that astroglia response could be involved in the RTP801-dependent cognitive alterations in the 5xFAD model.

Reinforcing this, genetic RTP801 inhibition in hippocampal $5 x F A D$ neurons had a general anti-inflammatory response, since it normalized in astrocytes the higher levels of GFAP but also the levels of RTP801 in comparison to WT. Although RTP801 expression in human and murine astrocytes has been described [60], its putative function in this cell type has never been investigated.

Astrocytes have emerged as key regulators of remote memory formation [61, 62] and molecular modifications in astroglial cells induce changes in rodent models of learning and memory [63]. Moreover, astrocytes are essential for the expression of synaptic plasticity phenomena such as long-term potentiation [64], displaying higher motility rates of their processes than dendritic spines [65]. Importantly, gliosis per se is strongly associated with cognitive decline $[66,67]$. Hence, the correction of neuroinflammatory events upon genetic inhibition of neuronal RTP801 could be enough to observe significant memory improvements in the 5xFAD model.

A major question from our results is how transducing hippocampal principal neurons corrects astrogliosis and microgliosis in the 5xFAD mice since it seems to be mTOR-independent. We found that silencing neuronal RTP801 in 5xFAD mice downregulated the levels of two inflammasome receptors, NLRP1 and NLRP3, and their effector, procaspase 1. This lines with the results obtained in macrophages, adipocytes, and macrophageadipocyte cocultures, where silencing RTP801/REDD1 diminished caspase 1 and NLRP3 levels as well as IL-1 $\beta$ secretion [53].

Since NLRP1 is mostly expressed in neurons [42], where the knockdown of RTP801 takes place and is sensitive to A [68], we speculate that neuronal RTP801 silencing reduces NLRP1 inflammasome which, in turn, would contribute to preventing the NLRP3-mediated inflammatory response in glia and the increase of RTP801 levels in astrocytes.

The lack of significant differences in IL-1 $1 \beta$ or cleaved caspase 1 suggests that RTP801 silencing could be affecting the priming activation step, which depends on gene expression of NLRP1, NLRP3 and the procaspase $1[69,70]$. On the other hand, procaspase 1 complexed with NLRPs in the absence of ASC can mediate other responses such as cell death [71]. This could become important in older 5xFAD mice where neuron death becomes evident (9-10 months old) [29], since silencing RTP801 seems to modulate the levels of procaspase 1.

More studies are warranted to understand the inflammatory crosstalk between neurons, microglia, and astrocytes over the timeline of the disease and how A $\beta$-induced neuronal RTP801 upregulation affects the other two cell types.

The advantage to target RTP801 levels, unlike mTOR activity, is that they are commonly upregulated in neurodegenerative conditions $[17,19,72]$. Therefore, its modulation could be more effective in a pathologic context of AD in comparison to targeting mTOR, with a wider and more complex spectrum of functions during the disease [73-75].

In summary, RTP801 is upregulated in AD mouse models and $A D$ brains, and normalizing its hippocampal expression in the 5xFAD model prevented the appearance of the inflammatory response and restored cognitive deficits. This work frames RTP801 as a promising biomarker and a new pharmacological target in AD.

\section{MATERIALS AND METHODS}

\section{Human postmortem samples}

Postmortem hippocampal samples from Alzheimer's disease patients and controls were obtained from Banc de Teixits Neurològics (IDIBAPS, Barcelona, Spain). The donation and obtaining of samples were regulated by the ethics committee of the Universitat de Barcelona. The sample processing followed the rules of the European Consortium of Nervous Tissues: BrainNet Europe II (BNEII). All the samples were protected in terms of individual donor identification following the BNEIl laws. Case information can be found in Supplementary Table 1. All the procedures for the obtention of postmortem samples followed the ethical guidelines of the Declaration of Helsinki and local ethical committees (Universitat de Barcelona ethical committee: IRB00003099).

\section{Animal models}

For this study, we used the transgenic mouse line 5xFAD (MMRRC catalog \#034840-JAX, RRID:MMRRC_034840-JAX). 5xFAD mice overexpress the 695amino acid isoform of the human amyloid precursor protein (APP695) carrying the Swedish, London, and Florida mutations under the control of the murine Thy- 1 promoter. Besides, they express human presenilin-1 (PSEN-1) carrying the M146L/L286V mutation, also under the control of the murine Thy-1 promoter [29]. We also used for biochemical studies male and female transgenic mice from the line CtTA/rTg4510 (IMSR Cat\# JAX:015815, RRID:IMSR_JAX:015815) expressing the P301L mutant variant of human four-repeat microtubule-associated protein Tau (0N4R tauP301L) at 6 months of age [33]. All animals were housed with access to food and water ad libitum in a colony room kept at $19-22^{\circ} \mathrm{C}$ and $40-60 \%$ humidity, under a 12:12-h light/dark cycle. Experimental animals were all males and used at 6 months of age and following the ethical guidelines (Declaration of Helsinki and NIH Publication no. 85-23, revised 1985, European Community Guidelines, and Spanish guidelines (RD53/2013) for handling animals and approved by the local ethical committee (University of Barcelona, 225/17 and Generalitat de Catalunya, 404/18).

\section{Tissue fixation and immunofluorescence}

Animals were euthanized by cervical dislocation. Left hemispheres were removed and fixed for 5 days in $4 \%$ paraformaldehyde in PBS. Free-floating coronal brain sections $(40 \mu \mathrm{m})$ were obtained using a Leica vibratome (Leica VT1000S). Sections were first washed twice in PBS-T (1× PBS $0.3 \%$ Triton X-100) and incubated in $50 \mathrm{mM} \mathrm{NH}_{4} \mathrm{Cl}$, twice for $15 \mathrm{~min}$. Blocking and permeabilization were performed for $1 \mathrm{~h}$ in PBS-T with $0.02 \%$ azide, $3 \%$ NGS, and $0.2 \%$ BSA. For amyloid plaque staining, blocking was performed for $4 \mathrm{~h}$, and blocking buffer contained $10 \%$ donkey serum in PBS $0.25 \%$ Triton X-100. Primary antibodies were diluted in blocking solution and incubated overnight at $4{ }^{\circ} \mathrm{C}$ in agitation. Secondary antibodies were diluted in blocking buffer and incubated for $2 \mathrm{~h}$ at room temperature. Nuclei were stained with Hoechst33342 (Thermo Fisher Scientific, \#H3570) diluted 1:5000 in PBS for $15 \mathrm{~min}$. Sections were washed with PBS-T between the different steps, and a final wash with PBS was performed prior to mounting with ProLong Gold Antifade Mountant. The following primary antibodies were used: anti-GFP chicken polyclonal $(1: 1000$, Synaptic systems, \#132006), anti-GFAP mouse monoclonal (1:1000, Sigma, \#G3893), anti-GFAP rabbit polyclonal (1:500, Dako, \#GA52461), anti-lba1 rabbit polyclonal (1:500, Wako, \#09-19741), anti-RTP801 rabbit polyclonal (1:100, Proteintech, \#10638-1-AP) and anti-APP (1:1000, Novus Biologicals, \#NBP2-62566). The following secondary antibodies were used: goat antichicken AlexaFluor488 (1:500, \#A11039), goat anti-mouse AlexaFluor555 (1:200, \#A21424), goat anti-mouse AlexaFluor647 (1:200, \#A21236), goat anti-rabbit AlexaFluor555 (1:200, \#A21429), goat anti-rabbit AlexaFluor647 (1:200, \#A21245) and donkey anti-rabbit AlexaFluor555 (1:600, \#A32794) (all from Thermo Fisher Scientific) (see Supplementary Table 2). Thioflavin $S$ (ThioS) staining was performed in free-floating sections following 
immunofluorescence procedure for APP and was performed as described in ref. [76]. Images were obtained with confocal microscopy (Zeiss LSM 880 and ZEN Software) at the Microscopy Service (Campus Clínic) with a $\times 10$, $\times 25$, or $\times 40$ magnification and standard (1 airy disc) pinhole (1 AU). Two sections from the dorsal hippocampus were analyzed per animal.

\section{Crude synaptosomal fractionation}

Crude synaptosomal fractions were isolated from both murine and human hippocampal brain samples. Samples were first homogenized in Krebs-Ringer (KR) buffer (125 mM NaCl, $1.2 \mathrm{mM} \mathrm{KCl}, 22 \mathrm{mM} \mathrm{NaHCO}$, $1 \mathrm{mM} \mathrm{NaH} \mathrm{PO}_{4}, 1.2 \mathrm{mM} \mathrm{MgSO}_{4}, 1.2 \mathrm{mM} \mathrm{CaCl}$, pH 7.4) supplemented with $10 \mathrm{mM}$ glucose, $0.31 \mathrm{M}$ sucrose (Sigma), and protease and phosphatase inhibitors (PhosSTOP and cOmplete, both from Roche, and PMSF, 1:100, from Sigma). Samples were centrifuged at $1.000 \times g$ for $10 \mathrm{~min}$ to discard debris. A sample of the resulting supernatant was kept as the Homogenate fraction. Next, the supernatant was centrifuged at $16.000 \times g$ for $15 \mathrm{~min}$ to obtain the cytosolic fraction and the crude synaptosomal fraction (resuspended in supplemented KR buffer).

\section{Western blotting}

Animals were euthanized by cervical dislocation. For western blot analyses, both hippocampi or entorhinal cortex were dissected out and stored at $-80^{\circ} \mathrm{C}$ until use. For AAV-injected 5xFAD mice, the dorsal and ventral hippocampus were dissected out separately. Samples (15-20 $\mu \mathrm{g})$ were resolved with NuPAGE ${ }^{\mathrm{TM}}$ Novex $^{\mathrm{TM}}$ polyacrylamide gels (3-8\% polyacrylamide gels with Tris-Acetate running buffer were used to analyze proteins with high molecular weight, while $12 \%$ and $4-12 \%$ polyacrylamide gels with MOPS SDS running buffer were used for proteins with small and intermediate weights, respectively). Proteins were transferred to nitrocellulose membranes with the iBlot2 system. All reagents and machinery were obtained from Thermo Fisher Scientific. Membranes were blocked with 5\% non-fat dry milk (Biorad) diluted in TBS-T (Tris-buffered saline containing $0.1 \%$ Tween-20). Primary antibodies were diluted in TBS-T with 5\% BSA (Sigma) and incubated overnight at $4{ }^{\circ} \mathrm{C}$ by shaking. The following primary antibodies were used (1:1.000 if not stated otherwise): anti-GFAP (Dako, \#GA52461), anti-GFP (Thermo Fisher Scientific, \#A-11122), anti-RTP801 (1:500, Proteintech, \#10638-1-AP), anti-Iba1 (Wako, \#019-19741), antiSynaptophysin (Synaptic Systems, \#101011), anti-TrkB (BD Biosciences, \#610102), anti-SV2a (Santa Cruz Biotech Technology, \#sc-376234), anti-PAkt Ser473 (Cell Signaling Technologies, \#4691), anti-Total Akt (Cell Signaling Technologies, \#4691), anti-P-S6 Ser235/236 ((Cell Signaling Technologies, \#4858), anti-Total S6 (Cell Signaling Technologies, \#2317), anti-P-mTOR Ser2448 (Cell Signaling Technologies, \#2971), anti-Total mTOR (Cell Signaling Technologies, \#2972), anti-NLRP1 (Novus, \#NBP1-54899), anti-NLRP3, anti-procaspase 1, anti-cleaved caspase 1, anti-cleaved IL-1 $\beta$, anti-ASC-TM1, anti-AIM2 (all from Cell Signaling Technologies, \#20836T), and anti- $\beta$-actin (Sigma, \#A3854). Mouse anti-actin primary antibody was already conjugated to horseradish peroxidase so it was incubated for $30 \mathrm{~min}$ before chemiluminescent protein detection. Anti-mouse and antirabbit secondary antibodies produced in goat and conjugated to HRP (Thermo Fisher Scientific, \#31460 and \#31430) were diluted 1:10.000 in blocking solution for $1 \mathrm{~h}$ at room temperature (summarized in Supplementary Table 3). Proteins were detected with Supersignal ${ }^{\mathrm{TM}}$ West Pico Plus chemiluminescent substrate (Thermo Fisher Scientific). Images were acquired with ChemiDoc ${ }^{\mathrm{TM}}$ (Bio-Rad) and quantified by densitometric analysis with ImageJ software $(\mathrm{NIH})$. When reincubation with another primary antibody was needed, membranes were washed with Restore Plus Western Blot Stripping buffer (Thermo Fisher Scientific) for $15 \mathrm{~min}$ to remove the previous signal.

\section{Behavioral tests}

Plus maze. The apparatus was made with two opposing $30 \times 8 \mathrm{~cm}$ open arms, and two opposing $30 \times 8 \mathrm{~cm}$ arms enclosed by $15 \mathrm{~cm}$-high walls placed $50 \mathrm{~cm}$ above the floor and dimly lit $(60 \mathrm{~lx})$. Each mouse was placed in the central square, facing an open arm, and the time spent in the open arms, which normally correlates with low levels of anxiety, was measured for $5 \mathrm{~min}$.

Passive avoidance. The passive avoidance (light-dark) paradigm was conducted in a 2-compartment box, where 1 compartment was dimly lit $(20 \mathrm{~lx})$ and the other brightly lit $(200 \mathrm{~lx})$. Both chambers were connected by a door $(5 \times 5 \mathrm{~cm})$. During training, mice were placed into the aversive brightly lit compartment, and upon entry into the preferred dimly lit compartment (with all four paws inside the dark chamber), they received a mild foot shock (2-s foot shock, $1 \mathrm{~mA}$ intensity). The latency of mice to enter into the dark chamber was recorded. Twenty seconds after receiving the foot shock, mice were returned to the home cage until testing $24 \mathrm{~h}$ later (long-term memory). For this retention test, mice were returned to the brightly lit compartment and the latency to enter the shock-paired compartment (dark chamber) was measured (10-min time cutoff).

Spontaneous alternation in a T-maze. The T-maze apparatus used was a wooden maze consisting of three arms, two of them situated at $180^{\circ}$ from each other, and the third representing the stem arm of the T, situated at $90^{\circ}$ to the other two. All arms were $45 \mathrm{~cm}$ long, $8 \mathrm{~cm}$ wide, and enclosed by a $20-\mathrm{cm}$ wall. The maze was thoroughly painted with waterproof gray paint. Light intensity was 5 lux throughout the maze. A starting area $(10 \mathrm{~cm})$ was located at the end of the stem arm and closed by a wooden guillotine door. Two identical guillotine doors were placed in the entry of the arms situated at $180^{\circ}$. The maze was elevated $60 \mathrm{~cm}$ above the floor. In the training trial, 1 arm was closed (novel arm) and mice were placed in the stem arm of the T (home arm) and allowed to explore this arm and the other available arm (familiar arm) for $10 \mathrm{~min}$, after which they were returned to the home cage. After $1 \mathrm{~h}$ (retaining test session), mice were placed in the stem arm of the T-maze and allowed to freely explore all three arms for $5 \mathrm{~min}$. The first choice to turn either to the familiar arm or to the new arm (alternation rate, \%) was monitored. In addition, the arm preference was also monitored and determined by calculation of the distance traveled in each arm $\times 100 /$ total distance traveled in both arms (familiar and novel).

Morris water maze. Spatial learning was assessed using a mouse-adapted Morris water maze (MWM) [77]. In a first phase to discard visual/physical deficiencies, each mouse underwent four trials in the water maze (circular pool; diameter: $100 \mathrm{~cm}$; height: $40 \mathrm{~cm}$, water depth: $25 \mathrm{~cm}$ ), with the visible platform and without extra maze distal cues. The escape platform $(10 \mathrm{~cm}$ diameter) was made visible by the attachment of a high-contrast striped flag. In the second phase (learning/acquisition phase), animals were trained 6 days, four trials per day. Four positions around the edge of the tank were arbitrarily designated north (N), south (S), east (E), and west (W) to provide four alternative start positions and define the division of the tank into four quadrants: NE, SE, SW, and NW. The platform was then submerged $1 \mathrm{~cm}$ below the water surface and placed at the midpoint of one of the quadrants. Each mouse was allowed to swim until they located and climbed onto the submerged platform. Mice that failed to locate the platform after $60 \mathrm{~s}$ were removed from the water and placed on the platform. At the end of the trial, all mice were left on the platform for $15 \mathrm{~s}$, before being returned to the home cage during the intertrial interval.

Grid test. For this test, mice were placed on a horizontal cage lid which was then agitated circularly three times and next turned upside down for $60 \mathrm{~s} 20 \mathrm{~cm}$ above a housing cage.

In all tasks, animal tracking and recording were performed using the automated SMART junior software (Panlab, Spain).

\section{Stereotaxic surgery and AAV transduction}

Following anesthesia with a mixture of $2 \%$ oxygen and isoflurane $(2 \%$ induction, $1.5 \%$ maintenance), we performed bilateral hippocampal injections of rAAV2/8-H1-shControl-RSV-GFP $\left(1.2 \times 10^{13} \mathrm{GCs}\right), \mathrm{rAAV} 2 / 8-\mathrm{H} 1-$ shRTP801-RSV-GFP $\left(1.07 \times 10^{13} \mathrm{GCs}\right)$ [19]. All the AAVs were purchased to the Unitat de Producció de Vectors from the Center of Animal Biotechnology and Gene Therapy at the Universitat Autònoma de Barcelona. We used the following coordinates (millimeters) from bregma (anteroposterior and lateral) and from the skull (dorsoventral); anteroposterior: -2.0 ; Lateral $+/-1.5$, and dorsoventral: -1.3 (CA1) and -2.1 (DG). The cannula was left to deliver $1 \mu \mathrm{l}$ of 1:1 virus in each depth for $4 \mathrm{~min}$, and two additional minutes were left to have complete virus diffusion. After $2 \mathrm{~h}$ of careful monitoring, mice were returned to their home cage for 3 weeks before starting the subsequent behavioral and biochemical analysis.

\section{Golgi staining and spine analysis}

Fresh brain hemispheres were processed with the FD Rapid GolgiStainTM kit (FD Neurotechnologies) as described in [78]. In all, 100- $\mu \mathrm{m}$ sections were obtained in a cryostat and mounted in gelatin-coated superfrost coverslips prior staining procedure. Brightfield images of impregnated apical dendrites from dorsal hippocampal CA1 pyramidal neurons were 
captured with a Leica AF6000 microscope (×63 magnification and 1.6 Zoom). Stacks were taken every $0.2 \mu \mathrm{m}$ and analyzed manually with FIJ software. Spine density was calculated in 20 proximal apical dendrites per animal, starting $5 \mu \mathrm{m}$ apart from the ramification and in segments with no overlap with other branches. Spine density values were averaged to obtain the mean for each animal. For a more precise description of dendritic spine shape, spine head diameter, and spine neck length were measured with FIJ and were analyzed as a continuous distribution (1200 dendritic spines were analyzed for each group). Image acquisition and analysis were performed blindly.

\section{Statistics}

Sample sizes were determined by using the power analysis method: 0.05 alpha value, 1 estimated sigma value, and $75 \%$ of power detection. All data are expressed as mean \pm SEM. Normal distribution was tested with d'Agostino and Pearson omnibus normality test. If the test was passed, statistical analysis was performed using parametric statistical analysis. Before pairs of comparisons, we performed the $F$ test to compare variances. In experiments with normal distribution statistical analyses were performed using the unpaired two-sided Student's $t$ test (95\% confidence) and the two-way ANOVA with the Bonferroni's or Tukey's post hoc tests as appropriate and indicated in the figure legends. $T$ test with Welch's correction was applied when variances were unequal. Values of $P<0.05$ were considered statistically significant. Correlation analyses were performed using Pearson. Kolmogorov-Smirnov test was used to analyze the distribution of dendritic spines' shape. Grubbs and ROUT tests were performed to determine the significant outlier values. All experiments in this study were blinded and randomized by blocks of animals. All mice bred for the experiments were used for pre-planned experiments and randomized to experimental groups. Data were collected, processed, and analyzed randomly. The experimental design and handling of mice were identical across experiments. Littermates were used as controls with multiple litters (3-5) examined per experiment.

\section{REFERENCES}

1. Bondi MW, Edmonds EC, Salmon DP. Alzheimer's disease: past, present, and future. J Int Neuropsychol Soc. 2017;23:818-31.

2. Long JM, Holtzman DM. Alzheimer disease: an update on pathobiology and treatment strategies. Cell. 2019;179:312-39.

3. Deture MA, Dickson DW. The neuropathological diagnosis of Alzheimer's disease. Mol Neurodegener. 2019;14. https://doi.org/10.1186/s13024-019-0333-5.

4. Tracy TE, Gan L. Tau-mediated synaptic and neuronal dysfunction in neurodegenerative disease. Curr Opin Neurobiol. 2018;51:134-8.

5. Mucke L, Selkoe DJ. Neurotoxicity of amyloid $\beta$-protein: synaptic and network dysfunction. Cold Spring Harb Perspect Med. 2012; 2. https://doi.org/10.1101/ cshperspect.a006338.

6. Hauss-Wegrzyniak B, Dobrzanski P, Stoehr JD, Wenk GL. Chronic neuroinflammation in rats reproduces components of the neurobiology of Alzheimer's disease. Brain Res. 1998;780:294-303.

7. Bales KR, Du Y, Holtzman D, Cordell B, Paul SM. Neuroinflammation and Alzheimer's disease: critical roles for cytokine/A $\beta$-induced glial activation, NF-KB, and apolipoprotein E. Neurobiol Aging. 2000;21:427-32.

8. Simón-Sánchez J, Schulte C, Bras JM, Sharma M, Gibbs JR, Berg D, et al. Genomewide association study reveals genetic risk underlying Parkinson's disease. Nat Genet. 2009:41:1308-12.

9. McGeer PL, McGeer EG, Yasojima K. Alzheimer disease and neuroinflammation. J Neural Transm Suppl. 2000;59:53-57.

10. Heneka MT, Carson MJ, Khoury JEL, Landreth GE, Brosseron F, Feinstein DL, et al. Neuroinflammation in Alzheimer's disease. Lancet Neurol. 2015;14:388-405.

11. Shoshani T, Faerman A, Mett I, Zelin E, Tenne T, Gorodin S, et al. Identification of a novel hypoxia-inducible factor 1-responsive gene, RTP801, involved in apoptosis. Mol Cell Biol. 2002;22:2283-93.

12. Ellisen LW, Ramsayer KD, Johannessen CM, Yang A, Beppu $H$, Minda $K$, et al. REDD1, a developmentally regulated transcriptional target of p63 and p53, links p63 to regulation of reactive oxygen species. Mol Cell. 2002;10:995-1005.

13. Brugarolas J, Lei K, Hurley RL, Manning BD, Reiling JH, Hafen E, et al. Regulation of mTOR function in response to hypoxia by REDD1 and the TSC1/TSC2 tumor suppressor complex. Genes Dev. 2004;18:2893-904.

14. Malagelada C, Ryu EJ, Biswas SC, Jackson-Lewis V, Greene LA. RTP801 is elevated in Parkinson brain substantia nigral neurons and mediates death in cellular models of Parkinson's disease by a mechanism involving mammalian target of rapamycin inactivation. J Neurosci. 2006;26:9996-10005.
15. Malagelada C, Zong HJ, Greene LA. RTP801 is induced in Parkinson's disease and mediates neuron death by inhibiting Akt phosphorylation/activation. J Neurosci. 2008;28. https://doi.org/10.1523/JNEUROSCI.3928-08.2008.

16. Romaní-Aumedes J, Canal M, Martín-Flores N, Sun X, Pérez-Fernández V, Wewering S, et al. Parkin loss of function contributes to RTP801 elevation and neurodegeneration in Parkinson's disease. Cell Death Dis. 2014;5:e1364.

17. Martin-Flores N, Romani-Aumedes J, Rue L, Canal M, Sanders P, Straccia M, et al RTP801 is involved in mutant huntingtin-induced cell death. Mol Neurobiol. 2015;53:2857-68.

18. Zhang Z, Chu S-F, Wang S-S, Jiang Y-N, Gao Y, Yang P-F, et al. RTP801 is a critical factor in the neurodegeneration process of A53T a-synuclein in a mouse model of Parkinson's disease under chronic restraint stress. $\mathrm{Br} J$ Pharm. 2018;175:590-605.

19. Martín-Flores N, Pérez-Sisqués L, Creus-Muncunill J, Masana M, Ginés S, Alberch J, et al. Synaptic RTP801 contributes to motor-learning dysfunction in Huntington's disease. Cell Death Dis. 2020;11:1-15.

20. Ota KT, Liu RJ, Voleti B, Maldonado-Aviles JG, Duric V, Iwata M, et al. REDD1 is essential for stress-induced synaptic loss and depressive behavior. Nat Med. 2014:20:531-5.

21. Ownby RL, Crocco E, Acevedo A, John V, Loewenstein D. Depression and risk for Alzheimer disease: Systematic review, meta-analysis, and metaregression analysis. Arch Gen Psychiatry. 2006;63:530-8.

22. Morel M, Couturier J, Pontcharraud R, Gil R, Fauconneau B, Paccalin M, et al. Evidence of molecular links between PKR and mTOR signalling pathways in $A \beta$ neurotoxicity: Role of p53, Redd1 and TSC2 Neurobiol. Dis. 2009;36:151-61.

23. Kim J-R, Lee S-R, Chung HJ, Kim S, Baek S-H, Kim JH, et al. Identification of amyloid beta-peptide responsive genes by CDNA microarray technology: involvement of RTP801 in amyloid beta-peptide toxicity. Exp Mol Med. 2003;35:403-11.

24. Damjanac M, Page G, Ragot $S$, Laborie G, Gil R, Hugon J, et al. PKR, a cognitive decline biomarker, can regulate translation via two consecutive molecular targets p53 and Redd1 in lymphocytes of AD patients. J Cell Mol Med. 2009;13:1823-32.

25. Canchi S, Raao B, Masliah D, Rosenthal SB, Sasik R, Fisch KM, et al. Integrating gene and protein expression reveals perturbed functional networks in Alzheimer's disease. Cell Rep. 2019;28:1103-1116.e4.

26. Yates SC, Zafar A, Hubbard P, Nagy S, Durant S, Bicknell R, et al. Dysfunction of the mTOR pathway is a risk factor for Alzheimer's disease. Acta Neuropathol Commun. 2013;1:3

27. Mecca AP, Chen MK, O'Dell RS, Naganawa M, Toyonaga T, Godek TA, et al. In vivo measurement of widespread synaptic loss in Alzheimer's disease with SV2A PET. Alzheimer's Dement. 2020;16:974-82.

28. Eng LF, Ghirnikar RS. GFAP and astrogliosis. Brain Pathol. 1994;4:229-37. https:// doi.org/10.1111/j.1750-3639.1994.tb00838.x.

29. Oakley H, Cole SL, Logan S, Maus E, Shao P, Craft J, et al. Intraneuronal $\beta$-amyloid aggregates, neurodegeneration, and neuron loss in transgenic mice with five familial Alzheimer's disease mutations: potential factors in amyloid plaque formation. J. Neurosci. 2006;26:10129-40.

30. Hongpaisan J, Sun MK, Alkon DL. PKC $\varepsilon$ activation prevents synaptic loss, $A \beta$ elevation, and cognitive deficits in alzheimer's disease transgenic mice. J. Neurosci. 2011;31:h 630-43.

31. Wu XM, Qian ZM, Zhu L, Du F, Yung WH, Gong Q, et al. Neuroprotective effect of ligustilide against ischaemia-reperfusion injury via up-regulation of erythropoietin and down-regulation of RTP801. Br J Pharm. 2011;164:332-43.

32. Yang EJ, Ahn S, Ryu J, Choi MS, Choi S, Chong YH, et al. Phloroglucinol attenuates the cognitive deficits of the 5XFAD mouse model of Alzheimer's disease. PLoS ONE. 2015;10. https://doi.org/10.1371/journal.pone.0135686.

33. Santacruz K, Lewis J, Spires T, Paulson J, Kotilinek L, Ingelsson M, et al. Medicine: Tau suppression in a neurodegenerative mouse model improves memory function. Science. 2005;309:476-81.

34. Giralt A, de Pins B, Cifuentes-Díaz C, López-Molina L, Farah AT, Tible M, et al. PTK2B/Pyk2 overexpression improves a mouse model of Alzheimer's disease. Exp Neurol. 2018;307:62-73.

35. De Pins B, Cifuentes-Díaz C, Thamila Farah A, López-Molina L, Montalban E, Sancho-Balsells $A$, et al. Conditional BDNF delivery from astrocytes rescues memory deficits, spine density, and synaptic properties in the 5xFAD mouse model of alzheimer disease. J Neurosci. 2019;39:2441-58.

36. Jawhar S, Trawicka A, Jenneckens C, Bayer TA, Wirths O. Motor deficits, neuron loss, and reduced anxiety coinciding with axonal degeneration and intraneuronal $A \beta$ aggregation in the 5XFAD mouse model of Alzheimer's disease. Neurobiol Aging. 2012;33:196.e29-196.e40.

37. Devi L, Ohno M. TrkB reduction exacerbates Alzheimer's disease-like signaling aberrations and memory deficits without affecting $\beta$-amyloidosis in 5XFAD mice. Transl Psychiatry. 2015;5:e562.

38. Connor B, Young D, Lawlor P, Gai W, Waldvogel H, Faull RLM, et al. Trk receptor alterations in Alzheimer's disease. Mol Brain Res. 1996:42:1-17. 
39. Cao T, Matyas JJ, Renn CL, Faden Al, Dorsey SG, Wu J. Function and mechanisms of truncated BDNF receptor TrkB.T1 in neuropathic pain. Cells. 2020;9. https://doi. org/10.3390/cells9051194.

40. Fenner BM. Truncated TrkB: beyond a dominant negative receptor. Cytokine Growth Factor Rev. 2012;23:15-24.

41. Hemonnot AL, Hua J, Ulmann L, Hirbec H. Microglia in Alzheimer disease: wellknown targets and new opportunities. Front Cell Infect Microbiol. 2019;9:233.

42. Kaushal V, Dye R, Pakavathkumar P, Foveau B, Flores J, Hyman B, et al. Neuronal NLRP1 inflammasome activation of Caspase-1 coordinately regulates inflammatory interleukin-1-beta production and axonal degeneration-associated Caspase6 activation. Cell Death Differ. 2015;22:1676-86.

43. Xiao NA, Zhang J, Zhou M, Wei Z, Wu XL, Dai XM, et al. Reduction of glucose metabolism in olfactory bulb is an earlier Alzheimer's disease-related biomarker in 5XFAD mice. Chin Med J. 2015;128:2220-7.

44. Quenon L, De Xivry JJO, Hanseeuw B, Ivanoiu A. Investigating associative learning effects in patients with prodromal Alzheimer's disease using the temporal context model. J Int Neuropsychol Soc. 2015;21:699-708.

45. Coughlan G, Laczó J, Hort J, Minihane AM, Hornberger M. Spatial navigation deficits-overlooked cognitive marker for preclinical Alzheimer disease? Nat Rev Neurol. 2018;14:496-506.

46. Kosaki Y, Lin T-CE, Horne MR, Pearce JM, Gilroy KE. The role of the hippocampus in passive and active spatial learning. Hippocampus. 2014;24:1633-52.

47. Brasted PJ, Bussey TJ, Murray EA, Wise SP. Role of the hippocampal system in associative learning beyond the spatial domain. Brain. 2003;126:1202-23.

48. Araya R, Vogels TP, Yuste R. Activity-dependent dendritic spine neck changes are correlated with synaptic strength. Proc Natl Acad Sci USA. 2014;111:E2895-E2904.

49. Adrian M, Kusters R, Storm C, Hoogenraad CC, Kapitein LC. Probing the interplay between dendritic spine morphology and membrane-bound diffusion. Biophys J. 2017; 113:2261-70.

50. Tønnesen J, Nägerl UV. Dendritic spines as tunable regulators of synaptic signals. Front Psychiatry. 2016;7:101

51. Lee DK, Kim JH, Kim WS, Jeoung D, Lee H, Ha KS, et al. Lipopolysaccharide induction of REDD1 is mediated by two distinct CREB-dependent mechanisms in macrophages. FEBS Lett. 2015;589:2859-65.

52. Yoshida T, Mett I, Bhunia AK, Bowman J, Perez M, Zhang L, et al. Rtp801, a suppressor of mTOR signaling, is an essential mediator of cigarette smokeinduced pulmonary injury and emphysema. Nat Med. 2010;16:767-73.

53. Pastor F, Dumas K, Barthélémy MA, Regazzetti $C$, Druelle N, Peraldi $P$, et al. Implication of REDD1 in the activation of inflammatory pathways. Sci Rep. 2017;7. https://doi.org/10.1038/s41598-017-07182-z.

54. Skendros P, Chrysanthopoulou A, Rousset F, Kambas K, Arampatzioglou A, Mitsios $A$, et al. Regulated in development and DNA damage responses 1 (REDD1) links stress with IL-1 $\beta$-mediated familial Mediterranean fever attack through autophagy-driven neutrophil extracellular traps. J Allergy Clin Immunol. 2017;140:1378-1387.e13.

55. Ferrer I, Marín C, Rey MJ, Ribalta T, Goutan E, Blanco R, et al. BDNF and full-length and truncated TrkB expression in Alzheimer disease. Implications in therapeutic strategies. J Neuropathol Exp Neurol. 1999;58:729-39.

56. Kemppainen S, Rantamäki T, Jerónimo-Santos A, Lavasseur G, Autio H, Karpova N, et al. Impaired TrkB receptor signaling contributes to memory impairment in APP/PS1 mice. Neurobiol Aging. 2012;33:1122.e23-1122.e39.

57. Holt LM, Hernandez RD, Pacheco NL, Torres Ceja B, Hossain M, Olsen ML. Astrocyte morphogenesis is dependent on BDNF signaling via astrocytic TrkB.T1. eLife 2019;8. https://doi.org/10.7554/eLife.44667.

58. Wu J, Renn CL, Faden Al, Dorsey SG. TrkB.T1 contributes to neuropathic pain after spinal cord injury through regulation of cell cycle pathways. J Neurosci. 2013:33:12447-63.

59. Matyas JJ, O'Driscoll CM, Yu L, Coll-Miro M, Daugherty S, Renn CL, et al. Truncated TrkB.T1-mediated astrocyte dysfunction contributes to impaired motor function and neuropathic pain after spinal cord injury. J Neurosci. 2017;37:3956-71.

60. Li Q, Cheng Z, Zhou L, Darmanis S, Neff NF, Okamoto J, et al. Developmental heterogeneity of microglia and brain myeloid cells revealed by deep single-cell RNA sequencing. Neuron. 2019;101:207-223.e10.

61. Gibbs ME, Hutchinson D, Hertz L. Astrocytic involvement in learning and memory consolidation. Neurosci Biobehav Rev. 2008;32:927-44.

62. Kol A, Adamsky A, Groysman M, Kreisel T, London M, Goshen I. Astrocytes contribute to remote memory formation by modulating hippocampal-cortical communication during learning. Nat Neurosci. 2020;23:1229-39.

63. Pinto-Duarte A, Roberts AJ, Ouyang K, Sejnowski TJ. Impairments in remote memory caused by the lack of Type 2 IP 3 receptors. Glia. 2019;67:glia.23679.

64. Henneberger C, Papouin T, Oliet SHR, Rusakov DA. Long-term potentiation depends on release of d-serine from astrocytes. Nature. 2010;463:232-6.

65. Haber M, Zhou L, Murai KK. Cooperative astrocyte and dendritic spine dynamics at hippocampal excitatory synapses. J Neurosci. 2006;26:8881-91.
66. Wan Y, Xu J, Meng F, Bao Y, Ge Y, Lobo N, et al. Cognitive decline following major surgery is associated with gliosis, $\beta$-amyloid accumulation, and $\tau$ phosphorylation in old mice. Crit Care Med. 2010;38:2190-8.

67. Sajja VSSS, Hlavac N, VandeVord PJ. Role of glia in memory deficits following traumatic brain injury: biomarkers of glia dysfunction. Front Integr Neurosci. 2016;10:7.

68. Tan MS, Tan L, Jiang T, Zhu XC, Wang HF, Jia CD, et al. Amyloid- $\beta$ induces NLRP1dependent neuronal pyroptosis in models of Alzheimer's disease. Cell Death Dis. 2014;5. https://doi.org/10.1038/cddis.2014.348.

69. Gritsenko A, Green JP, Brough D, Lopez-Castejon G. Mechanisms of NLRP3 priming in inflammaging and age related diseases. Cytokine Growth Factor Rev. 2020;55:15-25.

70. Herman FJ, Pasinetti GM. Principles of inflammasome priming and inhibition: Implications for psychiatric disorders. Brain Behav Immun. 2018;73:66-84.

71. Broz P, Von Moltke J, Jones JW, Vance RE, Monack DM. Differential requirement for caspase-1 autoproteolysis in pathogen-induced cell death and cytokine processing. Cell Host Microbe. 2010;8:471-83.

72. Malagelada C, Jin ZH, Jackson-Lewis V, Przedborski S, Greene LA. Rapamycin protects against neuron death in in vitro and in vivo models of Parkinson's disease. J. Neurosci. 2010;30:1166-75.

73. Paccalin M, Pain-Barc S, Pluchon C, Paul C, Bazin H, Gil R, et al. The relation between p70S6k expression in lymphocytes and the decline of cognitive test scores in patients with Alzheimer disease [1]. Arch Intern Med. 2005;165:2428-9.

74. Li X, Alafuzoff $\mathrm{I}$, Soininen $\mathrm{H}$, Winblad B, Pei JJ. Levels of mTOR and its downstream targets $4 \mathrm{E}-\mathrm{BP} 1, \mathrm{eEF} 2$, and eEF2 kinase in relationships with tau in Alzheimer's disease brain. FEBS J. 2005:272:4211-20.

75. An WL, Cowburn RF, Li L, Braak H, Alafuzoff I, Iqbal K, et al. Up-regulation of phosphorylated/activated p70 S6 kinase and its relationship to neurofibrillary pathology in Alzheimer's disease. Am J Pathol. 2003;163:591-607.

76. Ly PTT, Cai F, Song W. Detection of neuritic plaques in Alzheimer's disease mouse model. J Vis Exp. 2011;53:2831.

77. Giralt A, Saavedra A, Carretón O, Xifró X, Alberch J, Pérez-navarro E, et al. Increased PKA signaling disrupts recognition memory and spatial memory: role in Huntington's disease. Hum Mol Genet. 2011;20:4232-47.

78. Alvarez-Periel E, Puigdellívol M, Brito V, Plattner F, Bibb JA, Alberch J, et al. Cdk5 contributes to Huntington's disease learning and memory deficits via modulation of brain region-specific substrates. Mol Neurobiol. 2018;55:6250-68.

\section{ACKNOWLEDGEMENTS}

We thank Ana López (María de Maeztu Unit of Excellence, Institute of Neurosciences, University of Barcelona, MDM-2017-0729, Ministry of Science, Innovation, and Universities) for technical support and Dr. Núria Martín-Flores and Dr. Elena Ribé for helpful discussion.

\section{AUTHOR CONTRIBUTIONS}

LP-S, AS, JS, GC, ML-T, MAH, AG, and CM designed the experiments. LP-S, AS, JS, and GC performed the experiments and analyzed the data. LP-S, AS, JS, GC, AG, and CM interpreted the results and wrote the MS. ML-T, MAH, E-MM, and JA contributed to the MS writing and provided feedback and helpful discussion on the MS.

\section{FUNDING}

Financial support was obtained from the Spanish Ministry of Science, Innovation and Universities grants SAF2014-57160-R (AEI/FEDER, UE) and SAF2017-88812 R (AEI/ FEDER, UE) for CM, RTI2018-094678-A-I00 for AG, and SAF2017-88076 for JA. The project was supported in part by the Katharina-Hardt-Foundation to E-MM. A. Giralt is a Ramón y Cajal researcher (RYC-2016-19466). We thank Portal d'Avall S.L. for the L.PS. fellowship and support on this project.

\section{ETHICS STATEMENT}

All procedures were performed in compliance with the NIH Guide for the Care and Use of Laboratory Animals and approved by the local animal care committee of Universitat de Barcelona following European (2010/63/UE) and Spanish (RD53/2013) regulations for the care and use of laboratory animals. Human samples were obtained following the guidelines and approval of the local ethics committee (Hospital Clínic of Barcelona's Clinical Research Ethics Committee).

\section{CONFLICT OF INTEREST}

The authors declare no competing interests. 


\section{ADDITIONAL INFORMATION}

Supplementary information The online version contains supplementary material available at https://doi.org/10.1038/s41419-021-03899-y.

Correspondence and requests for materials should be addressed to A.G. or C.M.

Reprints and permission information is available at http://www.nature.com/ reprints

Publisher's note Springer Nature remains neutral with regard to jurisdictional claims in published maps and institutional affiliations.
Open Access This article is licensed under a Creative Commons Attribution 4.0 International License, which permits use, sharing, adaptation, distribution and reproduction in any medium or format, as long as you give appropriate credit to the original author(s) and the source, provide a link to the Creative Commons license, and indicate if changes were made. The images or other third party material in this article are included in the article's Creative Commons license, unless indicated otherwise in a credit line to the material. If material is not included in the article's Creative Commons license and your intended use is not permitted by statutory regulation or exceeds the permitted use, you will need to obtain permission directly from the copyright holder. To view a copy of this license, visit http://creativecommons. org/licenses/by/4.0/.

(c) The Author(s) 2021 